\title{
Deutsch als Fremdsprache und der Studienstand- ort Deutschland. Entwicklungslinien des Faches aus der Sicht (s)eines Verbandes ${ }^{1}$
}

\section{Armin Wolff; Dietrich Eggers; Rolf Ehnert; Klaus Kirsch}

\section{A) Der Arbeitskreis Deutsch als Fremdsprache beim DAAD von 1972-1989}

\section{Armin Wolff}

\subsection{Einleitung}

Für die 25. Jahrestagung des Arbeitskreises und späteren Fachverbandes Deutsch als Fremdsprache ist es sicher angebracht und richtig, Rückschau zu halten und aus der Sicht des Fachverbandes die Linien nachzuziehen, die die Entwicklung unseres Faches deutlich machen, und in Erinnerung zu rufen, mit welchen Vorstellungen, Motiven, Absichten und Zielen engagierte Fachleute für Deutsch als Fremdsprache Ende der 60er Jahre begannen, sich über das Fach Deutsch als Fremdsprache in seinem damaligen $\mathrm{Zu}-$ stand an den Hochschulen Gedanken zu machen.

An der Tatsache, daß für dieses Unterfangen vier Referenten aufgeboten wurden, mag man erkennen, daß es sich allein organisatorisch um ein merkwürdig kompliziertes Fach handelt, mit seinen Lehr- und Forschungs- gebieten an den Hochschulen und Fachhochschulen, den Studiengängen Deutsch als Fremdsprache (als volle Studiengänge, Teil-, Aufbau, Zusatzstudiengänge in allen Varianten vorhanden), mit seinen Studienkollegs, den Aufgaben der Mittlerorganisationen im In- und Ausland (wie z. B. DAAD und Goethe-Institut), den privaten Einrichtungen, die sich der Sprachvermittlung verschrieben haben. Wir wollen es wagen, dieses vielseitige Gebäude für Sie noch einmal entstehen zu lassen und dies aus der Perspektive und unter Darstellung der Aufgaben des Arbeitskreises Deutsch als Fremdsprache und späteren Fachverbandes Deutsch als Fremdsprache.

2.0 Das Fachstudium muß attraktiv sein, die deutsche Sprache ist es schon

Mit Überlegungen, wie das Fach Deutsch als Fremdsprache entwickelt werden sollte, wollten eben diese Fachleute schon zu Beginn der 70er Jahre ihren Beitrag dazu leisten, daß der Studienstandort Deutschland für ausländische Studienbewerber trotz der immer wieder als Hin-

1 Bei diesem Beitrag handelt es sich um die vier überarbeiteten Teilbeiträge des Eröffnungsvortrages für die 25. Jahrestagung Deutsch als Fremdsprache des Fachverbandes Deutsch als Fremdsprache (FaDaF) vom 22. bis 24 . Mai 1997 an der Johannes GutenbergUniversität Mainz. 
dernis gesehenen Sprachbarriere Deutsch attraktiv bleibt. Ich sehe das - wenn Sie erlauben - aus einer anderen Perspektive: Die deutsche Sprache ist auch in den zurückliegenden Jahren nicht anders, nicht schwieriger, nicht weniger schön geworden. Wenn sie heute - mehr als früher - zum Hindernis für Studierwillige aufgebaut wird, ist dies ein Operieren am falschen Gegenstand. Oder anders: Man schlägt den Esel und meint aber eigentlich den Herrn. Was meine ich damit: In unserer internationaler gewordenen Welt - wir nennen es heute gern Globalisierung - ist auch die Konkurrenzsituation schärfer geworden. Dies bedeutet, daß nicht das vermeintliche Hindernis deutsche Sprache angegangen werden muß. Die Hochschulen müssen mit ihren fachlichen Angeboten gegenüber ausländischen Studienbewerbern wieder in die Lage kommen, sich von Studien- und Abschlußangeboten in englisch- oder französischsprachigen Bereichen positiv abzuheben oder sich mit diesen mindestens vergleichen zu können. Nur durch eindeutige Leistungsangebote in den Fachdisziplinen ${ }^{1}$ und einem bereits bestehenden und anpassend auszubauenden Sprachlehrangebot Deutsch als Fremdsprache wird es gelingen, ausländische Studienbewerber an die deutschen Hochschulen zurückzuholen, die bei außergewöhnlichen fachlichen Angeboten das Hindernis Sprache wie früher auch - nicht nur bereitwillig in Kauf nehmen, sondern Deutsch als wichtige Kultursprache gerne mit nach Hause nehmen werden. Das Fachstudium muß also (wieder) attraktiver werden, die deutsche Sprache ist es schon.

\subsection{Warum mußte und konnte der AK- $\mathrm{DaF}$ gegründet werden?}

Wie hat das Ganze angefangen? Mit einer großen Windstille. Denn noch im Jahre 1970 ist im Bildungsbericht der Bundesregierung, in dem diese ihr bildungspolitisches Konzept darlegt, von Deutsch als Fremdsprache nicht die Rede, obwohl spätestens seit Anfang der 60er Jahre ein rapides Anwachsen der Zahl der ausländischen Studienbewerber und Studierenden und vor allem der ausländischen ArbeitnehmerInnen $\mathrm{zu}$ registrieren ist (Wierlacher 1975: 119).

Es bestand damals folgende Situation:

\subsection{Die wissenschaftliche Beschäfti-} gung mit Deutsch als einer Fremdsprache, seine Vermittlung, die Weiterentwicklung des Faches Deutsch als Fremdsprache, die Beschäftigung mit seiner Methodik und Didaktik, wurden einerseits von außen erzwungen durch die wachsende Zahl der ausländischen Studierenden. Sie brauchten, um hier ihr Studium erfolgreich absolvieren zu können, ausreichende Sprachkenntnisse, um möglichst rasch in den Wissenschaftsprozeß der Hochschulen integriert zu werden. Hinzu kam andererseits der Zwang von innen, nämlich die wachsende Zahl der in Deutschland lebenden ArbeitnehmerInnen und ihre Familien, die bei der sozialen und sprachlichen Orientierung und Integration in die deutsche Umwelt der Hilfe bedurften.

3.2 Deutschland ist in besonderer Weise auf internationale Kontakte angewiesen. Das Ausländerstudium, d.h. der längerfristige Aufenthalt von Ausländern zum

1 Dies hat z. B. der Ministerpräsident des Freistaates Bayern, Edmund Stoiber, am 6. Mai 1997 im Rahmen einer Veranstaltung an der Universität Regensburg gefordert. In ähnlicher Weise äußerte sich der Staatssekretär im Bayerischen Staatsministerium für Unterricht und Kultus, Wissenschaft und Kunst, Rudolf Klinger, anläßlich des diesjährigen DAAD-Stipendiatentreffens an der Universität Regensburg vom 9. bis 11. Mai 1997 
Zwecke der Ausbildung und des Studiums an unseren Hochschulen, ist von großer Bedeutung für unser Land. Bei aller Attraktivität des deutschen Hochschulsystems und der wissenschaftlichen Studien- und Forschungsmöglichkeiten, die wir in unserem Lande haben, tun die Verantwortlichen gut daran, sich um eine bessere Kompatibilität des deutschen Bildungssystems gegenüber englisch oder französisch geprägten Bildungssystemen $\mathrm{zu}$ bemühen und so die immer lauter beklagte rückläufige Internationalität unserer Hochschulen zu stoppen und positiv $\mathrm{zu}$ wenden. $\mathrm{Ob}$ allerdings das verstärkte Angebot englischsprachiger Studiengänge an deutschen Hochschulen der richtige Weg ist, den der DAAD mit seinem neuesten Aktionsprogramm vorschlägt, muß man zunächst sehr kritisch sehen, und dieser Weg bedarf erst noch eines überzeugenden Beweises für seine Richtigkeit.

3.3 Studien- bzw. Forschungswillige haben darüber hinaus eine Schwierigkeit zu überwinden: Sie müssen sich die nötigen Sprachkenntnisse aneignen, die den Studienerfolg in vertretbarer Studienzeit garantieren. Die Angebote der Lehrgebiete Deutsch als Fremdsprache und der Studienkollegs sind daher für eine erfolgreiche Integrationsphase und für einen erfolgreichen Verlauf des Hochschulstudiums ein unverzichtbarer Bestandteil des Ausländerstudiums, den es quantitativ und qualitativ zu erhalten, zu sichern und den sich verändernden Bedürfnissen angepaßt auszubauen gilt.

»Ich sehe die Berechtigung solcher studienvorbereitender und studienbegleitender Angebote durch die Hochschulen vor allem darin, daß ja ein solcher Sprachkurs nicht ziellos angeboten wird, sondern daß im Sprachkurs eine Orientierung angelegt ist, nämlich die Ausrichtung auf ein Fachstudium«. (Hansgert Schulte 1990: 15)
Dabei muß auch eine Überprüfbarkeit der studienqualifizierenden Sprachkenntnisse nach den für ein Hochschulstudium zu setzenden Anforderungen nach einheitlichen Kriterien gegeben sein.

Die Sprache des Gastlandes, in das ein Studienbewerber oder ein Forscher gehen, spielt für den Studien- bzw. Forschungserfolg eine zentrale Rolle. Diese sprachlichen Anforderungen in bezug auf den Wissenschaftsbetrieb klar vorzugeben und zu strukturieren, mußte geleistet werden. Die vielfältig gegebenen Hochschulverhältnisse mußten in organisatorisch-administrativer und fachbezogener Hinsicht transparent gemacht und koordiniert werden. Es mußte ein Instrument gefunden werden, das Hilfestellung leisten konnte bei der Bewältigung der Eingangsvoraussetzung Sprache, und zwar in einer für alle Hochschulen möglichst vergleichbaren Weise: Ein Arbeitskreis Deutsch als Fremdsprache drängte sich geradezu auf.

\subsection{Vom Arbeitskreis zum Fachverband Deutsch als Fremdsprache}

\subsection{Die Anfänge}

Von einer großen Windstille und den Mühen, die das Fach Deutsch als Fremdsprache hatte, um auf den Weg zu kommen, war die Rede. Um so erfrischender für uns alle klingt gegen Ende der 70er Jahre die Feststellung Harald Weinrichs während der 6. Jahrestagung Deutsch als Fremdsprache des AKDaF in Bonn beim DAAD, daß

»von einem leichten, angenehmen Rückenwind bewegt sich der Forschungs- und Lehrbereich Deutsch als Fremdsprache im letzten Jahrzehnt in das Gefüge der akademischen Disziplinen hineingeschoben hat» (Weinrich 1979: 17).

Dieser leichte, angenehme Rückenwind konnte nur etwas bewirken, weil er enga- 
gierte, ideenreiche Fachleute für Deutsch als Fremdsprache beflügeln konnte. Denn: Um etwas bewegen zu können, bedarf es nicht nur des frischen Windes, sondern insbesondere tatkräftiger Persönlichkeiten, die mit Initiativkraft und Umsicht Neues gestalten wollen und auch können.

In den Jahren 1971/72, nach Diskussionen insbesondere mit ebenso engagierten Leitern altehrwürdiger Akademischer Auslandsämter über den Stellenwert von Deutsch als Fremdsprache und seine organisatorische, fachlich richtige Einbindung in den Wissenschaftsbetrieb, entschlossen sich einige DaF-Lehrkräfte, das Aufgabenfeld Deutsch als Fremdsprache neu im akademischen Betrieb zu verankern (es bestand seinerzeit an den Hochschulen das Lehrgebiet Deutsch als Fremdsprache größtenteils als Verwaltungsreferat bei den Akademischen Auslandsämtern), und zwar möglichst als eigenständige Disziplin in der für eine qualifizierte Sprachausbildung zuständigen Fakultät der Hochschulen. Dies geschah 1972 im Rahmen der bis dahin vom Goethe-Institut durchgeführten jährlichen Tagung der Leiter der Lehrgebiete/ Lektorate Deutsch als Fremdsprache. Damit war der $\mathrm{AKDaF}$ geboren, dessen erste Mitglieder von 1972 auf 1973 das vorbereiteten, was aus dem Unternehmen AK$\mathrm{DaF}$ eine gesicherte Institution werden lassen sollte.

Sich aus der mütterlich-fürsorgenden Umgebung des Goethe-Instituts lösend, brach man auf, weil man sich erwachsen genug wähnte, eigene, selbständige Wege gehen zu können, beschloß während der letzten Tagung im Hause Goethe noch das »'Strukturmodell Lehr- und Forschungsgebiet Deutsch als Fremdspracher vom 25. Mai 1972« (Info DaF 1/1974: 1112) und machte sich damit auf den Weg, um für das neue Unternehmen AKDaF einen väterlich-beratenden Partner zu su- chen, der helfend, stützend und schützend zur Seite stehen konnte und wollte. Man fand ihn in Bonn beim DAAD, der aus seinem Selbstverständnis heraus den AKDaF beim DAAD in seinem Haus gerne aufnahm und ihm die notwendigen Mittel für seine Arbeit an die Hand gab und auch heute noch - unter völlig veränderten Bedingungen - bereitstellt.

»Die Unterstützung, die der DAAD dem Arbeitskreis Deutsch als Fremdsprache [...] leistet, entspringt dem Selbstverständnis des DAAD, nicht nur Förderungsprogramme für ausgewählte Gruppen von ausländischen und deutschen Hochschulangehörigen durchzuführen, sondern auch eine Gesprächsplattform zu bieten für Fragen des internationalen Hochschulaustausches, insbesondere des Ausländer- und des Auslandsstudiums«. (Schulte, Info DaF 1/1974)

Dieser AKDaF führte auf der Basis einer Verfahrensordnung seine Aufgaben durch und verstand sich als die auf der Jahrestagung gewählte Vertretung der Lehr- und Forschungsgebiete DaF der Hochschulen unter selbstverständlichem Einschluß der Lehrkräfte für Deutsch als Fremdsprache an den Studienkollegs.

\subsection{Das Strukturmodell}

Das Strukturmodell, mit dem man in den rauhen Wind der Wissenschaftslandschaft segelte, enthält alle grundlegenden Fragestellungen, die das Fach Deutsch als Fremdsprache beschäftigen und beschäftigen müssen:

- Es wird darin die Vernachlässigung des Faches beklagt (wir sehen uns - trotz aller Fortschritte und Erfolge - immer wieder gezwungen, diese Vernachlässigung zu beklagen, weil es ja nicht nur leichte, beflügelnde Rückenwinde gibt, sondern uns immer wieder auch heftige Gegenwinde ins Gesicht blasen);

- die zentrale Aufgabe der (einzurichtenden) Lehr- und Forschungsgebiete DaF an den Hochschulen wird definiert (und wir tun auch heute gut daran, diese im- 
mer wieder einmal an den tatsächlichen Gegebenheiten zu überprüfen): Integration der ausländischen Studierenden und Wissenschaftler in den gesellschaftlichen und fachwissenschaftlichen Kommunikationsprozeß, und zwar über eine angemessene, durch Forschung fundierte Sprachvermittlung;

- eingeschlossen sind hierbei geeignete Meßmethoden und Überprüfungsverfahren (bis heute ist ein zentraler Diskussionspunkt das Prüfungswesen DaF);

- und es wurden Ausbildungsaufgaben im Bereich DaF postuliert und deren Umsetzung gefordert.

\subsection{Die Aufgaben des Arbeitskreises}

Der AKDaF hat seine Arbeit im wesentlichen auf fünf Bereiche konzentriert, die bereits weitestgehend im Strukturmodell vorgegeben wurden:

\subsubsection{Hochschulpolitische Vertretung}

Seine wichtigste Aufgabe war und ist in erster Linie eine hochschulpolitische: Die Lehrgebiete Deutsch als Fremdsprache brauchten eine Vertretung in hochschulpolitischen Fragen im Innenverhältnis zu den Hochschulen, aber auch nach außen. Er hat sich daher stets darum bemüht, gute Kontakte zu allen DaF-bezogenen Institutionen herzustellen und mit ihnen im Dia$\log$ zu bleiben. Beteiligungen an Tagungen - wie der GAL und der IDT - gehören zum selbstverständlichen Tätigkeitsfeld des AKDaF genauso wie auf Deutsch als Fremdsprache bezogene Stellungnahmen, wie z. B. 1983 die Stellungnahme zum Bericht der Bundesregierung zur Situation der deutschen Sprache in der Welt. In gleicher Weise hat sich der AKDaF bereits 1974 darum bemüht, Mitglied im Internationalen Deutschlehrerverband (IDV) zu werden, was allerdings erst 1993 realisiert werden konnte.

\subsubsection{Das Lehrangebot der Hochschulen}

Bei allen Aktivitäten des AKDaF war zu verdeutlichen, daß die Hochschulen gerade durch ihre Lehrgebiete Deutsch als Fremdsprache und die kontinuierliche Entwicklung des Faches Deutsch als Fremdsprache einen wesentlichen Beitrag zur Vermittlung und Förderung der deutschen Sprache leisten. Sichtbarster Ausdruck dieser Bemühungen ist nach dem Strukturmodell die Erarbeitung des »Rahmenplans Deutsch als Fremdsprache für >Sprachlehrveranstaltungen vor Aufnahme des Fachstudiums< und für studienbegleitende Lehrveranstaltungen an den Universitäten und Hochschulen der Bundesrepublik Deutschland «, der einen Orientierungsrahmen anbietet für diese Lehrveranstaltungen und sie in einer im Wissenschaftsbereich gebotenen Weise transparent macht. Er orientiert sich an den Bedürfnissen der Lerner und schafft damit Voraussetzungen, die eine Ungleichbehandlung der Adressaten möglichst gering halten sollen. Wichtig war dabei die Praxisbezogenheit unseres Faches und seine wissenschaftliche Konturierung auf breiter Grundlage in den Fachrichtungen Linguistik, Sprachlehr- und Lernforschung, Literatur- und Kulturwissenschaft, Wissenschaftstheorie und Didaktik.

Parallel dazu hat sich der AKDaF Gedanken machen müssen über eine qualifizierte und angemessene Aus- und Weiterbildung der im Bereich Deutsch als Fremdsprache tätigen Lehrkräfte. Er hat diesem Thema 1981 eine ganze Tagung gewidmet und hat »Leitlinien der Lehrerausbildung im Bereich Deutsch als Fremdsprache entwickelt« (Wolff 1982: 67-76).

\subsubsection{Vergleichbare Prüfungsbedingungen}

Der AKDaF hat erhebliche Anstrengungen unternommen, eine Annäherung und Vereinheitlichung bei den Anforderungen für die Sprachprüfung zu erreichen. Er entwickelte 1972 die »Rahmenordnung 
für die Prüfung zum Nachweis deutscher Sprachkenntnisse (PNdS) für ausländische Studienbewerber an den Wissenschaftlichen Hochschulen der Bundesrepublik Deutschland einschließlich Berlin (West)«, die am 12. Dezember 1972 von der 101. WRK beschlossen und den Hochschulen zur Umsetzung empfohlen wurde. Diese PNdS-Ordnung wurde inzwischen noch zweimal geänderten Bedingungen und Entwicklungen angepaßt, wobei für die Fassung der PNdS-Rahmenordnung 1983 und noch viel stärker bei der seit 1. Juni 1996 geltenden Rahmenordnung für die Deutsche Sprachprüfung für ausländische Studienbewerber (DSH) immer stärker darauf geachtet wurde, daß die Prüfungsanforderungen, wie sie allgemein gelten sollten, an den Hochschulen und den Studienkollegs für das Fach Deutsch als Fremdsprache in gleicher Weise realisiert werden können. Gerade mit der neuen DSH erfolgte eine Öffnung in der Weise, daß kooperativ durchzuführende DSH-Prüfungen auch im Ausland möglich sind. Wichtig in diesem Zusammenhang ist auch die Weiterentwicklung von Fragen des Prüfungswesens, wie sie eine von der Kultusministerkonferenz eingerichtete sog. Expertenrunde als Auftrag hat. In dieser Expertenrunde sind Lehrgebiete und Studienkollegs auf Ebene der Hochschulrektorenkonferenz zusammengeführt mit dem Goethe-Institut und der Zentralstelle für das Auslandsschulwesen, um gemeinsam an der weiteren Vergleichbarkeit der verschiedenen DaF-Prüfungen $\mathrm{zu}$ arbeiten.

\subsubsection{Die Tagungen}

Der AKDaF schuf für das Fach mit großer Hilfe durch den DAAD eine Plattform für wissenschaftliche Diskussionen. Er bereitet vor, organisiert und veranstaltet - in Zusammenarbeit mit einer jeweils gastgebenden Hochschule - seit 1973 regelmäßig die Jahrestagung Deutsch als Fremdsprache.
Man kann sicher feststellen, daß sich die Jahrestagungen im Laufe der Jahre zu einer zentralen und für die Fachwelt unverzichtbaren Veranstaltung entwickelt haben. Dies ist nicht nur so, weil wir an den Teilnehmerzahlen zu diesen Tagungen ein meist konstantes oder sogar steigendes Interesse feststellen, dies ist vor allem deshalb so, weil der AKDaF sich von Anfang an bemüht hat, mit in der Regel vier Themenbereichen für jede Tagung Diskussionsmöglichkeiten für Grundsatzfragen zu bieten, aktuelle methodische und didaktische Fragen zur Diskussion zu stellen, Theorie und Praxis unseres Faches immer wieder einer kritischen Betrachtung zu unterziehen. Ergänzend richtete der $\mathrm{AKDaF}$ 1985 erstmals in Saarbrücken das Forum Deutsch als Fremdsprache ein, eine Möglichkeit, sog. graue Materialien vorzustellen. Dieses Forum DaF ist auch für unsere Fachverlage (neben der Buchausstellung im Rahmen der Tagungen) eine Möglichkeit, verlagsneue und im Entstehen begriffene Lehrbücher einer interessierten Fachöffentlichkeit erstmals zu präsentieren. Es muß hier darauf verzichtet werden, die Themenbereiche aufzulisten, mit denen sich unsere Jahrestagungen von Erlangen 1973 bis Mainz 1997 beschäftigt haben. Es wären immerhin fast 100 (natürlich sich teilweise wiederholende) Themenschwerpunkte zu nennen.

Neben den Angeboten der jeweiligen Jahrestagungen ergab sich die Notwendigkeit, Spezialthemen in Fachtagungen anzubieten und diskutieren zu lassen. Der AKDaF hat dieses Angebot von Fachtagungen, die ihre Themen oft aus den Diskussionen während der Jahrestagungen gewannen, ebenso zu einer ständigen (möglichst jährlich ein- bis zweimal anzubietenden) Einrichtung werden lassen wie die Jahrestagung selbst. Themen zur Methodik und Didaktik, zur Fachsprache, zur Phonetik, zur Literatur und Landeskunde und zu Fragen der 
Prüfungen waren beispielsweise Inhalte derartiger fachlicher Angebote ${ }^{1}$.

\subsubsection{Die Publikationsmöglichkeiten}

"Allein die Diskussion der Verfahrensordnung durch das Plenum der Jahrestagung 1973 hat gezeigt, wie wünschenswert und notwendig eine Rückkopplung des Arbeitskreises zum Plenum der Jahrestagung einerseits und zu den Lehrkräften im Bereich Deutsch als Fremdsprache andererseits ist. Die gemeinsame Aufgabe aller Lehrkräfte in diesem Bereich macht es notwendig, zur Verbesserung der Koordination und Kooperation den Informationsaustausch zwischen den einzelnen Hochschulen und den anderen in gleicher Weise betroffenen Institutionen zu verbessern «. (Wolff/Hoffmann/ Rug, Info DaF 1/1974: 2)

Damit war die Zeitschrift Info DaF geboren, und zwar zunächst als Informationsblatt für den Bereich Deutsch als Fremdsprache, als Forum begründeter Meinungen und als Informationsinstrument, durch das über den weiteren Auf- und Ausbau von Deutsch als Fremdsprache berichtet werden konnte. Daß sich daraus einmal eine der wichtigsten Fachzeitschriften für Deutsch als Fremdsprache entwickeln würde, war damals noch nicht zu erkennen. Immerhin erscheint Info DaF bereits im 24. Jahr mit 6 Heften jährlich. Eine Besonderheit dieser Zeitschrift ist das Doppelheft 2/3 eines Jahrgangs, das zunächst am Lehrgebiet Deutsch als Fremdsprache der Universität Mainz als Projekt für den Arbeitskreis entwickelt auch heute noch von hier aus betreut wird: Gemeint ist die »Kommentierte Auswahlbibliographie von Neuerscheinungen für das Fach Deutsch als Fremdsprache«. Es dürften über die Jahre hinweg ca. 3000 Rezensio- nen und Besprechungen sein, die auf diese Weise für unser Fach in Info DaF gesammelt wurden.

Mit der Entwicklung zur Fachzeitschrift, die der DAAD in Zusammenarbeit mit dem AKDaF herausgibt, hatte sich der AK$\mathrm{DaF}$ unversehens seines Mitteilungsblattes beraubt. Es mußt hierfür Ersatz geschaffen werden, zunächst in Form des Rundbriefes und seit Einrichtung der Geschäftsstelle des FaDaF in Münster in Gestalt der Informationszeitschrift FaDaF Aktuell ${ }^{2}$.

Entscheidend für einen erfolgreichen Studienverlauf ist für ausländische Studienbewerber bei der Unterschiedlichkeit der einzelnen Hochschulangebote und bei der Vielzahl von zu bewältigenden administrativen, organisatorischen und studienrelevanten Bedingungen und Voraussetzungen, daß sie schon in ihrem Heimatland klare und überschaubare Informationen zur Verfügung haben. Für unser Fach hat sich der AKDaF gemeinsam mit dem DAAD darum bemüht, indem er in mehrfachen Auflagen die Sprachlehrangebote und die Studiengänge Deutsch als Fremdsprache dokumentiert und zuletzt die DSH-Broschüre erstellt hat.

Mit den Materialien Deutsch als Fremdsprache mit inzwischen 46 Heften hat sich der $\mathrm{AKDaF} / \mathrm{FaDaF}$ eine Publikationsreihe geschaffen, in der seit 1975 nicht nur die Jahrestagungen dokumentiert werden und die Ergebnisse der Fachtagungen bekannt gemacht werden können. Sie dient auch als Publikationsmöglichkeit für Unterrichts- und Prüfungsmaterialien, enthält inzwischen eine Reihe von Dissertationen und Zusammenfassungen zu angefertigten Magisterarbeiten (Anlage 3).

1 Eine Übersicht über die Themenschwerpunkte der 25 Jahrestagungen und der Fachtagungen, die der $\mathrm{AKDaF} / \mathrm{FaDaF}$ durchgeführt hat, ist diesem Beitrag als jeweilige Anlage beigegeben. Siehe Seite 578-582.

2 Geschäftsstelle des Fachverbandes Deutsch als Fremdsprache, Hüfferstr. 27/I, 48149 Münster. FaDaF Aktuell erscheint dreimal jährlich als Verbandszeitschrift und dient als Informationsinstrument für die Mitglieder des FaDaF. 


\section{B) Der Fachverband Deutsch als Fremdsprache von 1989-1997}

\section{Dietrich Eggers}

Nahezu zwei Jahrzehnte lang war der Arbeitskreis Deutsch als Fremdsprache ein Arbeitskreis beim DAAD. Dies hatte vor allem den Vorteil finanzieller Absicherung bei gleichzeitiger Entlastung von Sekretariatsaufgaben. Allerdings war die Feinabstimmung zwischen Arbeitskreis und DAAD nicht immer leicht, und so wuchs auf beiden Seiten die Einsicht, daß die Gründung einer Gesellschaft oder eines Verbandes die einzig sinnvolle Fortentwicklung sei.

Die Gründung des Fachverbands erfolgte 1989. Auf der Verbandsebene ergaben sich dadurch weitere Möglichkeiten der wissenschaftlichen Zusammenarbeit und Auseinandersetzung mit anderen nationalen Verbänden: Im Hochschulbereich mit der Gesellschaft für Angewandte Linguistik, im Bereich der Lehreraus- und -weiterbildung mit dem Fachverband Moderne Fremdsprachen. Konsequenterweise führte dies dazu, daß der Fachverband Deutsch als Fremdsprache nun als selbständige, eigenständige Gesellschaft 1993 Mitglied des Internationalen Deutschlehrerverbandes wurde, was weitere Aktivitäten im Prozeß des wissenschaftlich-didaktischen Austausches auch über die Grenzen hinweg möglich werden läßt.

Der Verband öffnete sich dann auch auBeruniversitären Anbietern von Deutsch als Fremdsprache gegenüber. Die Interessengemeinschaft Qualitätskriterien Deutsch als Fremdsprache gründete 1996 einen Verein, dessen Aufgabe es ist, Institute, die dies wünschen, durch einen neutralen Beirat überprüfen zu lassen und ihnen bei positivem Urteil für einen mittelfristigen Zeitraum ein Qualitätsmerkmal zu bescheinigen.
In diese Zeit der Umstrukturierung fiel das Ereignis der deutschen Einheit. Ein reger Gedankenaustausch setzte ein anläßlich vieler Einzeltreffen, Jahrestagungen und Fachtagungen. Die Mitgliederzahl des Fachverbands stieg sprunghaft an. Dann allerdings folgte bald die Phase, in der deutlich wurde, daß nur eine vergleichsweise geringe Zahl von Kolleginnen und Kollegen auf feste Stellen übernommen wurde und das Unwort "abgewickelt « - und dies auf Personen bezogen - machte die Runde.

$\mathrm{Zu}$ Beginn der 90er Jahre suchte man im Bildungssektor generell nach einer Neubestimmung. Auch der Fachverband Deutsch als Fremdsprache bezog für seinen Bereich Position und formulierte zwanzig Thesen, die 1995 als Dresdner Erklärung von der Mitgliederversammlung der Jahrestagung in Dresden verabschiedet wurden.

Bei diesen Thesen handelt es sich nicht um eine wissenschaftsbezogene Selbstdarstellung eines Verbandes, sondern um Einsichten und Forderungen, an deren politischer und hochschulpolitischer Umsetzung wir noch arbeiten müssen.

Die ersten fünf Thesen zielen ab auf $»$ Die deutsche Sprache und ihre Bedeutung in der Welt«. Festgestellt wird, daß Deutsch ebenso Kultur- wie Wissenschaftssprache ist, Lehrgebiete sowohl die Kultursprache Deutsch als auch die Wissenschaftssprache Deutsch zu vermitteln haben.

»Die Deutschen sollten ein bewußteres und loyaleres Verhältnis zu ihrer Sprache entwickeln, um sie als internationale Verkehrssprache zu gebrauchen und zu institutionalisieren.«

So lautet die These zwei, und Lothar Wittmann, Leiter der Kulturabteilung im Auswärtigen Amt, der diese These zitiert, fügt hinzu: »Ich möchte ergänzen: Wer die eigene Sprache verleugnet, überzeugt keinen Partner in der Welt «. In These fünf wird die Forderung erhoben, 
»das Bewußtsein zu stärken, daß Deutsch eine international gepflegte Publikationssprache und internationale Kongreßsprache ist " (Wittmann 1996: 69). Wie notwendig diese Forderung ist, belegt die Tatsache, daß anläßlich der internationalen Studentenmesse 1996 in Brüssel der Vertreter des Fachverbands laut Vorgabe des DAAD seinen Beitrag auf Englisch halten sollte, was gleichermaßen zu Protesten der Teilnehmer als auch der in Brüssel arbeitenden Simultandolmetscher führte, so daß der Kollege dann doch Deutsch sprechen konnte.

Der nächste Themenkomplex der Dresdner Erklärung befaßt sich mit der »wissenschaftlichen Forschung und Ausbildung in Deutsch als Fremdsprache«. Gefordert wird:

»Bestehende Einrichtungen zur Erforschung und Vermittlung des Deutschen als Fremdsprache an den Hochschulen zukunftsorientiert auszubauen und - wo sie fehlen - neu zu schaffen « (7. These).

Gefordert wird weiterhin, daß »DaF-Lehrkräfte ihrer Qualifikation entsprechend eingesetzt und [...] nach bundesdeutschen Maßstäben vergütet werden« (11. These). Zu erläutern gilt in diesem Zusammenhang, daß $60 \%$ aller Lehrveranstaltungen im Inland über Zeitverträge durch Lehrbeauftragte angeboten werden, ein Tatbestand, der dem Industrieland Deutschland, das um eine Neubestimmung des Studienstandorts bemüht ist, $\mathrm{zu}$ denken geben müßte. Deswegen beziehen sich die Thesen des dritten Teils gezielt auf die Vermittlung des Deutschen als Fremdsprache an Hochschulen und fordern, daß die Integrationsphase, also die Phase vor Aufnahme des Fachstudiums, wie auch die Begleitphase während des Studiums flächendeckend in allen Bundesländern angeboten werden (12. These).

Die abschließenden Thesen beinhalten Forderungen an staatliche Institutionen, den "Studienaufenthalt ausländischer
Studierender « dadurch zu erleichtern, daß bürokratische, aber auch soziale und materielle Hürden beseitigt werden.

Mir kommt die Aufgabe zu, den Zeitraum 1989 bis heute zu umreißen. Somit sei auch die »Deutsche Sprachprüfung für ausländische Studienbewerber « erwähnt, die als Rahmenordnung 1996 von der Hochschulrektorenkonferenz und der Kultusministerkonferenz verabschiedet wurde und bis Ende Mai 1997 von den Hochschulen umgesetzt werden muß. Diese Sprachprüfung - sie löst die seit 1972 bzw. 1983 gültige Prüfung zum Nachweis deutscher Sprachkenntnisse ab - gewährt den Lehrgebieten und Studienkollegs einen größeren Handlungsspielraum, setzt zum anderen jedoch einen intensiven kritischen Erfahrungsaustausch zu Prüfungsaufgaben voraus, um die Gleichwertigkeit der Prüfungen zu gewährleisten. Der DAAD hat eine Broschüre über diese neue Prüfungsordnung mit mehreren Prüfungsbeispielen veröffentlicht und wird sie weltweit verteilen. Lassen Sie mich schließlich noch auf eine Entwicklung eingehen, die einem Paradigmawechsel gleichkommt. Ende der 80er Jahre begannen die ERASMUS-Programme zu greifen. Westeuropäische Studenten kamen in der Regel für zehn Monate an bundesdeutsche Hochschulen; viele konnten sofort mit Erfolg ihr Grundstudium fortsetzen, für andere waren Intensivkurse Deutsch und studienbegleitende Lehrveranstaltungen dringend erforderlich, um den Studienerfolg nicht $\mathrm{zu}$ gefährden. Im akademischen Jahr 1996 wurden an der Universität Mainz 650 ERASMUS-Studenten immatrikuliert, von denen etwa die Hälfte an Sprachlehrveranstaltungen Deutsch als Fremdsprache teilnahm. Dieser sprunghafte Anstieg stellte uns - wie auch viele andere Lehrgebiete - vor schwer lösbare Probleme, zumal auf Grund der geringen Verweildauer diese Gruppe nicht in die 
auf die Sprachprüfung vorbereitenden Kurse integriert werden konnte.

Was bedeutet diese Entwicklung für die Lehrgebiete Deutsch als Fremdsprache? Sie müssen - wie auch bisher - ausländische Studierende während der sprachlichen Integrationsphase auf die Deutsche Sprachprüfung vorbereiten, zum anderen muß ein Kurssystem für europäische SOKRATES-Studenten, wie das Programm ab 1997 heißt, ab 1998 gehören auch Studierende der MOE-Staaten dazu, konzipiert und angeboten werden, ein Kurssystem, das auf Grund der vorgegebenen Programmstruktur andere Komponenten ausweisen muß. Das Kursangebot für SOKRATES-Studenten muß sich sowohl auf Deutsch als Kultur- als auch auf Deutsch als Wissenschaftssprache erstrecken. Wegen der zeitlichen Begrenztheit müssen Lehrgebiete Sprachlehrkerne erarbeiten, die zu sprachstandsangemessenen fachorientierten Sprachmodulen spezifiziert werden können. Sprachmodule müssen auf Vernetzbarkeit hin angelegt sein und die Nutzung interaktiver Medien mit einbeziehen. Während ihres Aufenthalts in Deutschland sollte es SOKRATES-Studenten ermöglicht werden, unterhalb der DSH-Ebene ein Universitätszertifikat Deutsch zu erwerben.

SOKRATES-Partner sollten bei der Erstellung des Studienplans studienbegleitende Sprachlehrveranstaltungen mit berücksichtigen. Bei der Vergabe von Kreditpunkten - dies wird in Deutschland nicht nach dem anglo-amerikanischen System, sondern nach Kriterien des Arbeitsaufwandes und Arbeitsvolumens erfolgen - ist darauf zu achten, daß die Partnerhochschulen innerhalb des Anrechnungssystems ECTS (European Credit Transfer System) Anrechnungspunkte vorsehen. Wenn dies geschieht, aber nur dann, hat die deutsche Hochschule die Pflicht, diese Lehrveranstaltungen anzubieten, was für die Lehrge- biete Chance und Herausforderung zugleich bedeutet.

Die Lehrgebiete Deutsch als Fremdsprache müssen, wie dies z. B. in Mainz der Fall ist, in den SOKRATES- und ECTSGremien vertreten sein, um an diesen Veränderungen mitwirken zu können.

Wir müssen - jenseits des kommerziellen Marktes - ein modulares System von Sprachlehrmaterialien erstellen, ein Projekt, das der Fachverband koordinieren sollte, denn es kann nur hochschulübergreifend angegangen werden.

Wir brauchen gezielt ausgebildete Sprachlehrerinnen und Sprachlehrer, die nicht nur Allgemeinsprache, sondern vor allem fächerübergreifende und fachbezogene Wissenschaftssprache vermitteln können. Wir benötigen für diesen Aufgabenbereich dringend Stellen; denn die Teilnehmerzahl hat sich verdoppelt, während in anderen Fächern ein Rückgang von Studentenzahlen festzustellen ist. Und, falls kurzfristig nicht mit Stellen zu rechnen ist, benötigen wir erhebliche Finanzmittel, um durch ein adäquates Sprachlehrangebot den Veränderungen im Hochschulbereich gerecht zu werden.

Die Kultusministerkonferenz hat sich in dem im März 1997 erschienenen Papier »Hochschulen und Hochschulpolitik vor neuen Herausforderungen « zur Standortfrage Deutschland geäußert, auch zur Studiensituation, zur modularen Struktur des Lehrangebots, zum Credit-Transfer-System. Diese Bereiche sollten in der Tat rasch geklärt werden, damit »deutsche Hochschulen für ausländische Wissenschaftler und Studenten attraktive Orte wissenschaftlicher Tätigkeit« bleiben oder es wieder werden (KMK Dokumentation 3/1997: 3).

Die Seitenhiebe, die bei der Frage des Studienstandorts Deutschland auf die Lehrgebiete niedergehen, empfinde ich als unnötig. Weder ist Deutsch so viel schwerer zu lernen als eine andere Spra- 
che, noch wurde durch die Rahmenordnung für die Deutsche Sprachprüfung eine zu hohe Hürde geschaffen (wir können nachweisen, was geschieht bzw. nicht geschieht, wenn Studierende mit geringer Sprachkompetenz in Vorlesungen sitzen oder an Seminaren teilnehmen), auch ist aus der Sicht der Sprachlehrforschung und der Fremdsprachendidaktik Deutsch so gut wie jede andere moderne Fremdsprache $\mathrm{zu}$ vermitteln, noch sind es die Lerninhalte selbst, die so abschreckend wirken könnten. Hier irrt der Präsident des DAAD, wenn er, wie kürzlich, gegenüber einer überregionalen Zeitung, die ihn wörtlich zitiert, sagt: »Wer bei uns im Grundstudium Physik belegen will und den Abschluß in seinem Heimatland erwerben will, muß nicht unbedingt die Meerseburger Zaubersprüche im Original lesen können«. (Berchem, SZ Nr. 15, 20.1.1997: 34) ${ }^{1}$. Recht hat er, nur liegt er in der Sache falsch, denn die Lehrgebiete vermitteln kein Althochdeutsch. Bei der Diskussion über das Aktionsprogramm des DAAD zur Förderung von Ausländern an deutschen Hochschulen sollte der Fachverband kritischer Partner bleiben, was den Bereich »Hilfen zur Bewältigung der sprachlichen Anforderungen « betrifft.

Unser aller Anliegen sollte die Pflege des Ausländerstudiums sein, das ja gesetzlich verankert ist. So heißt es z. B. in $\S 2$ des Hochschulgesetzes von Rheinland-Pfalz:

"Die Hochschulen fördern die internationale, insbesondere die europäische Zusammenarbeit im Hochschulbereich und den Austausch zwischen deutschen und ausländischen Hochschulen; sie berücksichtigen die besonderen Bedürfnisse ausländischer Studierender.« (Universitätsgesetz §2(6): 14)

\section{C) Einige (persönliche) Anmer- kungen zu den Studiengängen Deutsch als Fremdsprache}

\section{Rolf Ehnert}

1972 wurde, nach zahlreichen konzeptuellen Überlegungen, nach viel inneruniversitärem Widerstand auch, in Heidelberg ein wohlbegründeter Studiengang Deutsch als Fremdsprache eingerichtet, zu dem aber bis heute nur Ausländer zugelassen werden.

Bitte erlauben Sie mir eine kurze autobiographische Bemerkung; ich denke, daß ich gerade auch wegen meines Werdegangs eingeladen wurde, hier zu den Studiengängen Deutsch als Fremdsprache Stellung zu nehmen:

Nach zwei Jahren als assistant in Frankreich war ich neun Jahre lang Lektor an einer finnischen Universität, hatte aber schon vorher, während des Studiums, ausländische Studierende, zunächst - aus dem Rückblick - sehr dilettantisch, und dann schon ein wenig kompetenter ausländische Kinder und Jugendliche unterrichtet. Als ich 1975 nach Deutschland zurückkehrte, mit 15jähriger Erfahrung in einem »Beruf «, den ich nie erlernt hatte, war es schon verwunderlich, daß sich seit der Einrichtung des Heidelberger Studiengangs in Sachen Ausbildung von DaF-Lehrkräften nichts getan hatte. (In der DDR sah das ja anders aus.) So begann der damalige Arbeitskreis Deutsch als Fremdsprache (AKDaF) mit seiner Bonner Jahrestagung diesbezügliche Überlegungen, die langsam aufkeimten, $\mathrm{zu}$ beobachten und voranzutreiben. 1976 stellte ich an meiner Universität einen Antrag auf die Einrichtung eines Magisterstudiengangs, der 1979 anlaufen

1 Zitiert aus einer Glosse: »Campus: Überflüssige Zaubersprüche«, in: Süddeutsche Zeitung Nr. 15 vom 20.1.1997, 34. 
konnte. Unmittelbar danach übernahm Harald Weinrich den Lehrstuhl an der Münchener Universität.

Seither ging die Entwicklung schnell voran: Es wurden Voll- und Teilstudiengänge, Lehrstühle geschaffen. Nach den letzten Bestandsaufnahmen von 1994 (Henrici / Koreik) existieren bei 19 Vollstudiengängen (nach Krumm 1994: 13) 18 Lehrstühle. Studiengänge, die, mit einigen Varianten, den Namen Deutsch als Fremdsprache tragen, gibt es lediglich sechs: Bayreuth, Bielefeld, Heidelberg, Jena, Leipzig, München. In Bremen gibt es einen Lehramtsstudiengang. Die übrigen 13 betten Deutsch als Fremdsprache in einen anders benannten Studiengang, oft in einen germanistischen, ein. Daneben gibt es 31 Aufbau-, Ergänzungs-, Erweiterungsoder Zusatzstudiengänge, zusammen also 50 Studiermöglichkeiten.

Trotz der temperamentvollen Auseinandersetzung um die Konzeption des Faches, gerade in den allerletzten Jahren, hat sich seit Heidelberg, Bielefeld und München nichts wesentliches daran geändert, daß die Inhalte des Studiums, wie bei anderen Fremdsprachenphilologien auch, linguistische, landeskundliche und literaturwissenschaftliche, literaturgeschichtliche sind. Sie gruppieren sich, mit unterschiedlichen Gewichtungen, um den meist lehr-/lernwissenschaftlichen Kern der Lehre und Forschung. Gerade weil die Diskussion so rege ist, durch eine Reihe von Beiträgen in der Zeitschrift Deutsch als Fremdsprache, eine Monographie (Henrici/Koreik) und zwei Themennummern der Zeitschrift Germanistische Linguistik wohl dokumentiert und leicht zugänglich ist, will ich über die unterschiedlichen Standpunkte hier nichts sagen. Vielmehr möchte ich versuchen, mit wenigen Sätzen eine kleine Bilanz zu ziehen über die Leistungen des Faches, und in Form eines Ausblicks ge- genwärtige Schwierigkeiten und daraus resultierende Aufgaben skizzieren.

Die frühen Arbeiten zur Konstitution des Faches z. B. von Dietrich (1975), Wierlacher (1975), Weinrich (1979) sind dabei ebenso zu nennen wie die Etablierung einer Lehrwerkforschung (Mannheimer Gutachten), die Entwicklung von Übungstypologien, Prüfungen und Prüfungsordnungen. Es entstehen neun Einführungen in das Studium, das Jahrbuch Deutsch als Fremdsprache, zwei umfassende Handbücher, eine kaum mehr $\mathrm{zu}$ überblickende Fülle von Lehrbüchern und Lehrmaterialien, vier bedeutende Zeitschriften, eine sonst wohl nirgendwo existierende kommentierte Jahresbibliographie von Neuerscheinungen, eine umfangreiche Diskussion über Fachsprachen - ich beschränke mich auf nur dieses - und eben eine fundierte, theorie-praxisgeleitete, anwendungsbezogene, mit intensiven Praktika verbundene Ausbildung von inzwischen zahlreichen Lehrkräften.

Es kann nicht genug betont werden, wie wichtig für diese Ausbildung die Verzahnung mit der Sprachlehre ist, mit den PNdS/DSH-Sprachlehrangeboten an den Universitäten und den Volkshochschulen, für Aussiedler und anderswo: Hospitations- und Praktikumsmöglichkeiten für die Studierenden, die ihrerseits Tutoren für die ausländischen Studienbewerber sind, die wiederum als Probanden der Spracherwerbsforschung zur Verfügung stehen und als lebendige Vertreter ihrer jeweiligen Kulturen die Internationalität und Interkulturalität des Faches garantieren. Aus dem gleichen Grunde ist es auch so wichtig, daß möglichst viele ausländische Studierende in den Studiengängen eingeschrieben sind. Betrachtet man die Ausschreibungstexte des DAAD für Lektorate, so ist seit vielen Jahren Unterrichtserfahrung eine Voraussetzung für die Bewerbung. Schaut man sich die Voraussetzungen für die einzelnen Lektorate und die Aufgabengebiete 
näher an, so entdeckt man nahezu immer eine kaum erklärbare Diskrepanz:

Aufgaben: Sprachunterricht, Übersetzungsübungen, Landeskunde.

Voraussetzungen: Studienabschluß in Germanistik im Hauptfach; Lehrerfahrungen in DaF.

Aufgaben: Fremdsprachendidaktik, Übersetzung, Computerlinguistik, Landeskunde. Voraussetzungen: Studienabschluß in Germanistik im Hauptfach (Schwerpunkt: Linguistik).

Aufgaben: Sprachunterricht, Fachsprache Wirtschaft, Landeskunde.

Voraussetzungen: Studienabschluß Germanistik (DaF)

(Ausschreibungen für 1996)

Ich frage: Welche Germanistin, welcher Germanist hat in seinem Studium etwas erfahren über Fremdsprachenunterricht, Übersetzungstheorie und -technik, Translatorik (was zugegebenermaßen in den DaF-Studiengängen auch kaum vorkommt), Landeskunde, Fachsprachen und Wirtschaftswissenschaften?

Das Fach Deutsch als Fremdsprache hat sich im Bewußtsein der sog. Auslandsgermanistik, von löblichen Ausnahmen abgesehen, kaum durchgesetzt. Griechenland erkennt die deutschen Magisterabschlüsse nicht an. Es besteht weiterhin die Vorstellung, eine Lektorin, ein Lektor könne nur ein Germanist sein, in der Mehrzahl der Fälle sogar mit literaturwissenschaftlicher Schwerpunktbildung. Die Zentralstelle für das Auslandsschulwesen/die Kultusministerkonferenz entsendet keine Magister nach Mittel- und Osteuropa usw.

Hinzu kommt die mißliche Stellensituation, die fehlende soziale Absicherung, ja bereits Probleme mit der Berufsbezeichnung: Fremdsprachenlehrerin, Dozentin für Deutsch als Fremdsprache...? Nach wie vor scheinen höchstens ein Drittel aller in dem weiten Unterrichtsfeld Tätigen feste Stellen zu haben. In manchen Ländern werden die gut ausgebildeten Fachkräfte als bedrohliche Konkurrenz der einheimischen Lehrenden empfunden.

Dabei sind die Anforderungen dank der bekannten weltweiten Veränderungen etwa gegenüber meiner Lektorentätigkeit vor 25 Jahren in Finnland gestiegen insofern, als sowohl neue Kenntnisse und Fertigkeiten, aber auch Eigenschaften und Fähigkeiten notwendig geworden sind - und die AbsolventInnen der Studiengänge erfüllen sie!

Trotz dieser Professionalisierung sieht die Situation für Studienabgänger so aus wie geschildert. Aus Briefen von Absolventinnen:

»Im allgemeinen muß ich zugeben, daß die Perspektiven für DaF-AbsolventInnen meines Wissens nicht besonders gut sind. Kleine Honorarverträge und Unsicherheit sind an der Tagesordnung. Aufgrund der sozialen Kürzungen, die die Regierung anstrebt, werden immer weniger Gelder für den Unterricht mit Ausländern ausgegeben. Das bekomme ich jetzt sehr deutlich zu spüren, denn ich arbeite für den Sprachverband, der bereits sehr drastisch zu kürzen versucht.

Außerdem werden ständig neue Leute aus den unterschiedlichsten Berufen für den Deutschunterricht mit Ausländern rekrutiert. Das habe ich während einer Fortbildung im Goethe-Institut gehört. Sogar Leute, die nur das Abitur haben, werden zu DeutschlehrerInnen fortgebildet. Das trägt meiner Meinung nach zu einer Verminderung des Ansehens dieses Faches bei. Vielleicht sollte man den wissenschaftlichen Charakter des Studiums mehr betonen, Didaktik und Sprachlehrforschung stärker miteinander verbinden. Außerdem sollte Deutsch als Fremd-/Zweitsprache an den Schulen angeboten werden, das würde viele Vorteile mit sich bringen, vielleicht sollte man sich mehr in diesem Bereich engagieren. In Nordrhein-Westfalen würde vielleicht so ein Engagement Früchte tragen. Ich denke, als DaF-Absolventin ist es ziem- 


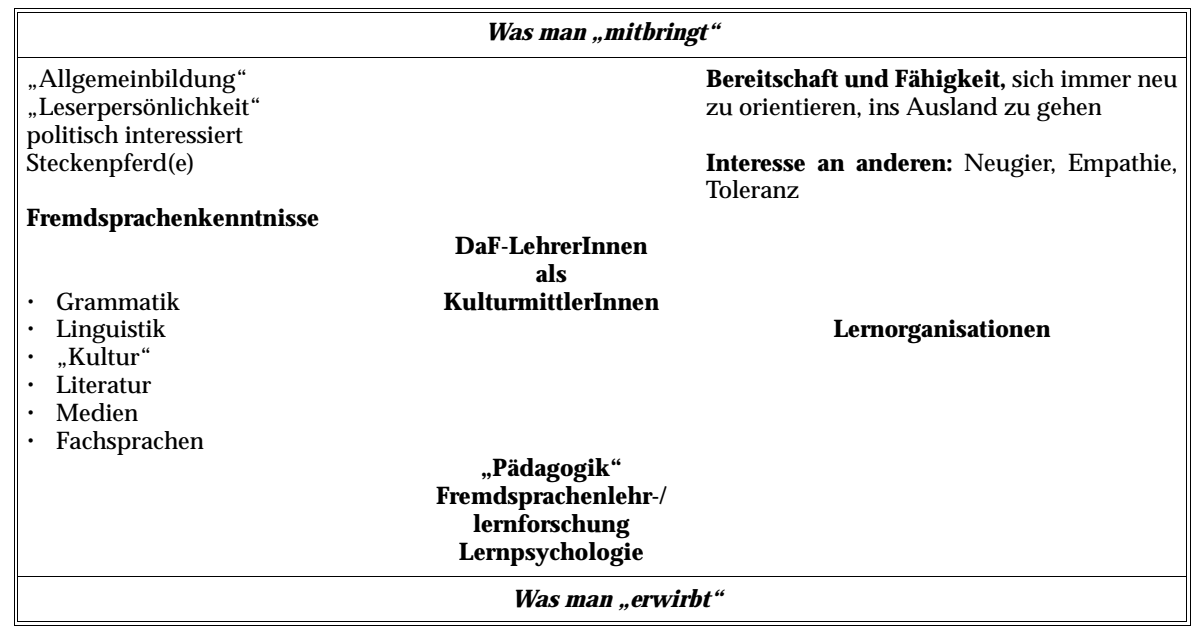

lich schwierig, eine Stelle zu finden, die mit diesem Beruf in Zusammenhang steht."

»Seit ersten Januar diesen Jahres arbeite ich als freie Lektorin an der Universität ... Die Aufgabenbereiche: Hörverstehen, Sprechtraining, Wirtschaftsdeutsch, Anfängerkurse für Nicht-Germanisten, Telefonkonversation. Die Bezahlung entspricht meiner vorherigen Arbeit [2000 DM netto, RE], wobei letztere aber nur 15 Stunden à $60 \mathrm{~min}$ umfaßt (plus Vorbereitungszeit, Erstellung von Skripts, Aufnahme von Fernsehsendungen...).

Eine interessante Tätigkeit, die aber leider aufgrund einer französischen Regelung auf zwei Jahre begrenzt ist. Nach Ablauf dieses Limits ist auch keine neue Anstellung an einer anderen Universität möglich. Einzige Chance: ein über den DAAD vermitteltes Lektorat - eine Hürde, die ich trotz meiner Abschlußnote $(1,0)$ bisher nicht zu überspringen vermochte. Ein Grund für das bisherige >Scheitern $<$ : fehlende Literaturkenntnisse meinerseits und unzureichende Toleranz gegenüber >neuen` didaktischen Ansätzen von seiten des französischen Auswahlgremiums. Es gilt eben, die Tradition des französischen Bildungssystems zu respektieren (auch in meiner aktuellen Tätigkeit), was zuweilen allem bisher Gelernten und selbst Entwickelten widerspricht.«
»Bisher habe ich leider keinen festen Job gefunden. Ich halte mich mit diversen Honorar- und Aushilfsjobs (DaF-Unterricht und auch anderes) über Wasser. Ich habe mich zwar recht viel beworben (überregional, allerdings nicht im Ausland), aber bislang ohne Erfolg. Ich bin noch nicht mal eine Runde weitergekommen.

Inzwischen überlege ich, eine andere Ausbildung $\mathrm{zu}$ machen, da meine derzeitige Qualifikation offensichtlich völlig unbrauchbar ist. Dies ist allerdings finanziell recht schwierig, da man als Hochschulabsolventin durch alle Maschen des sozialen Netzes fällt.

Im Moment bin ich der Ansicht, daß der Studiengang DaF in bezug auf unsere Berufsaussichten nicht funktioniert - leider.«

Die Curricula müssen ständig überdacht und nach Möglichkeit angepaßt, die Fächerkombinationen müssen noch erweitert werden.

Absolventen werden wie die nahezu aller anderen Fächer in eine Berufswelt entlassen, die dem herkömmlichen Bild in nichts mehr entspricht. Der Hamburger Freizeit- und Zukunftsforscher Horst W. Opaschowski stellte kürzlich fest:

»... Das Normalarbeitsverhältnis stirbt. Schon heute leisten mehr als zwei von fünf Beschäftigten $(43,5 \%)$ regelmäßig oder gele- 
gentlich Schicht-, Nacht- und Wochenendarbeit. Und jedes dritte Arbeitsverhältnis in Deutschland dauert kein ganzes Jahr, garantiert also auch kein kontinuierliches Einkommen mehr. Immer mehr Personen sind in Teilzeitverhältnissen beschäftigt oder gehen einer sozialversicherungsfreien Beschäftigung nach.

Die Arbeit für die Fulltime-Jobber wird immer intensiver und konzentrierter, zeitlich länger und psychisch belastender, dafür aber auch - aus der Sicht der Unternehmen - immer produktiver und effektiver. Dieser Minderheit der Fulltime-Jobber steht in Zukunft die Mehrheit der Gelegenheitsarbeiter mit schlecht bezahlten Teilzeit- und Mehrfachjobs gegenüber, die sich fast ein ganzes Leben lang mit dem Phänomen der Unterbeschäftigung und Unterbezahlung arrangieren müssen."(Unveröffentlichter Vortrag vor der Handwerkskammer Bielefeld)

Epilog: Am Rande der Tagung wurde mir vorgeworfen, daß ich die Studierenden eines bestimmten Aufbaustudienganges bitter enttäuscht habe.

\section{D) Ausländerstudium in Deutschland im Wechselspiel von Angebot und Nachfrage}

\section{Klaus Kirsch}

Es gehört zur Redekultur solcher Veranstaltungen wie dieser, daß man sich feiert oder feiern läßt, Verdienste und Leistungen Revue passieren läßt und sich gegenseitiger Zustimmung in der - womöglich noch besseren - Fortsetzung des Erreichten versichert. Erlauben Sie mir einen prosaischeren Beitrag zu diesem Anlaß, und gestatten Sie mir einige Überspitzungen, die kritische Absicht und Kürze meines Beitrages nahelegen.

Aus einer bildungspolitischen Nische und hochschulspezifischen Marginalie will mir scheinen - ist das Ausländerstudium seit wenigen Jahren ins Licht einer breiteren Öffentlichkeit getreten. »End- lich, richtig «, möchte man applaudieren, wenn nicht die Motive dieser Öffentlichkeitsverstärkung - pädagogisch gesprochen - extrinsisch anmuten würden.

Schauen Sie mit mir auf die Sprache der »Reformpapiere «:

Die gemeinsame Erklärung der Regierungschefs von Bund und Ländern zur Steigerung der internationalen Wettbewerbsfähigkeit des Studienstandorts Deutschland vom Dezember 1996 stellt fest, »[...] in Deutschland ausgebildete Entscheidungsträger sind angesichts $z u$ nehmender Globalisierung in Wirtschaft, Wissenschaft und Gesellschaft zentrale Mittler und Kooperationspartner «.

Die »internationale Konkurrenz« und Stand der Lehre und Forschung an deutschen Hochschulen habe zu einer Einbuße an Attraktivität bei dynamischen Schwellenländern Asiens und Lateinamerikas geführt.

Die Verbesserung der internationalen Wettbewerbsfähigkeit der deutschen Hochschulen sei zentrale hochschulpolitische Aufgabe. »Der Ausländeranteil von 4,1\% an den Studierenden in der BRD [...] ist steigerungsfähig." »Marketing [...] im Ausland «, »regelmäßige Präsentation deutscher Hochschulen auf Fachmessen und Tagungen im Ausland müssen von Bund und Ländern unterstützt werden«. Im Bericht der Kultusministerkonferenz vom 18.11.96 wird immerhin, bei den »Wettbewerbsfaktoren " des Studienstandortes, die »deutsche Sprache als Grundlage für Wissensvermittlung an deutschen Hochschulen« genannt. Die AG >Bewertung der KMK schreibt am 11./12.03.97, »Überlegungen zum >Standort< Deutschland seien nicht allein ausschlaggebend [...] für die Höherbewertung « ausländischer Bildungsnachweise.

Die Wortwahl dieser Zitatenlese mit fachspezifischer Terminologie der Wirtschaft legt den Verdacht nahe, daß wirtschaftliche Interessen fast wichtiger $\mathrm{zu}$ sein 
scheinen als sachbezogene Reforminteressen. Ist vielleicht das eherne Marktgesetz von Angebot und Nachfrage, in unserem Falle die mangelnde Nachfrage nach Studienplätzen durch potentielle Kundenländer, Motiv einer Studienreform? Nicht von ungefähr werden die Wachstumsregionen Südostasiens und Lateinamerikas immer wieder hervorgehoben, deren künftige Eliten man an deutschen Hochschulen ausbilden möchte. Es geht bei der Reform des Ausländerstudiums offenbar nicht um ausländische Studenten schlechthin, sondern um die bestimmter Weltregionen; nicht um alle Zielgruppen, sondern um die Eliten; nicht um Studienanfänger - wie andere Papiere belegen -, sondern vor allem um Graduierte und Postgraduierte. Diese ausländischen Studenten sollen der BRD Gewinn bringen, aber möglichst wenig kosten. Sprachausbildung und Studienvorbereitung in Deutschland sollen möglichst minimiert werden; denn die überproportional langen Studienzeiten in Deutschland sollen zunächst einmal dadurch verkürzt werden, daß der direkte Hochschulzugang für ausländische Studenten erweitert wird und die Sprachausbildung in Deutschland verkürzt wird, wie aus der AK >Bewertung der KMK zu hören und in einem ihrer Papiere zu lesen ist.

Daraus läßt sich unschwer erkennen, daß diese Reformankündigungen nicht alle Fragen beantworten, sondern viele Fragen nach Zielen und Chancen des Ausländerstudiums in Deutschland offen lassen:

- Sind die gewünschten Eliten ausländischer Studienbewerber durch Liberalisierung der Studien- und Sprachvorbereitung nach Deutschland zu lenken?

- Genügt das Angebot an Deutschkursen im Ausland den quantitativen und qualitativen Erfordernissen einer Studienvorbereitung?
- Wie steht es um die Realisierung der Studienvorbereitung im Ausland, wenn Goethe-Institute geschlossen werden, deren Zertifikate aber andererseits obligatorische Voraussetzung für die Erteilung von Studentenvisa sind?

- Sind die Sprachkursangebote an deutschen Hochschulen ausreichend?

- Sind die bisherigen studienbegleitenden Maßnahmen der Hochschulen geeignet, das Ausländerstudium in Deutschland attraktiver zu machen?

- Sind internationale Titel, kürzere Ausbildungszeiten, englischsprachige Studienanteile ausreichende Zielsetzungen einer Reform des Ausländerstudiums in Deutschland?

- Wo bleiben bei diesen Reformüberlegungen die Inhalte, Studienorganisation, die Lehr- und Prüfungsverfahren?

Lassen Sie mich bei meinen kritischen Fragen bitte auch das eigene Denken und Handeln nicht ausnehmen und auf seine Sachdienlichkeit und Reformtauglichkeit befragen.

- Ist eine einheitliche Rahmenordnung eine Garantie auch für einheitliche Anforderungen und Standards?

- Haben nicht Teilnehmerzahlen - die Betonung liegt auf Zahlen - auch für Sprach- und Vorbereitungskurse an den Lehrgebieten und Studienkollegs eine Bedeutung sui generis, weil Zahlen über Stellen und Stellen über Finanzmittel entscheiden?

- Beeinflussen nicht oft auch hier Kriterien von Angebot (an Bewerbern) und Nachfrage (nach Bewerbern) Anforderungsniveau und Bewertungsspielräume?

- Ist sprachliche Vorbereitung der jetzigen Art noch die sachgerechte Vorbereitung auf ein Fachstudium von kürzerer Dauer als bisher?

Die Fragen zu reformrelevanten Problemen lassen sich fortsetzen.

Der Sinn dieser Sequenz könnte sein, vor kurzschlüssiger, monokausaler Problem- 
bewältigung $\mathrm{zu}$ warnen. Wenn jetzt in Gremien wie Rektorenkonferenz, Konferenz der Ministerpräsidenten, der KMK, der Bundesregierung nach Lösungen gesucht wird, um das Ausländerstudium in Deutschland attraktiver $\mathrm{zu}$ machen, ist dies zunächst uneingeschränkt zu begrüßen. Wenn aber - wie es aus diesen Gremien raunt - die Lösung der Probleme vor allem in formalen, administrativen Maßnahmen gesehen wird und die Sachkriterien aus dem Blick geraten sollten, dann wird dem Ausländerstudium in Deutschland ein fragwürdiger Dienst erwiesen.

Ein Reformhandeln, das z. B. durch Änderung der Zeugnisbewertung, durch Analogie der Schuljahre, Wochenstunden und durch die damit gegebene Vermehrung der Bewerberzahlen für den deutschen Hochschulzugang, durch formale Angleichung an internationale Studienpraxis die Probleme des Ausländerstudiums zu lösen glaubt, erinnert an das »Reformhandeln « nach dem sogenannten »Sputnikschock « vor über 30 Jahren.

In der damaligen oft nur quantitativen Mobilisierung der Bildungsreserven durch formales Reformhandeln scheinen noch heute viele Probleme der Hochschulen begründet. Es ist keine Therapie von Symptomen, sondern im Sinne unserer Arbeit und unter unserer selbstkritischen Mitarbeit eine Reform an Haupt und Gliedern nötig!

Ich wünsche mir und uns allen, daß wir den Mut haben mögen, das Ausländerstudium nicht nur nach Marktgesetzen, sondern Reformen inhaltlicher, sachdienlicher Art mit langem Atem zu fördern.

\section{Literatur}

Albers, Hans Georg; Herwig, Oliver; Tanzer, Harald (Zusammenstellung): »Deutsch als Fremdsprache an außeruniversitären Institutionen in Deutschland «. Hrsg. vom Deutschen Akademischen
Austauschdienst in Zusammenarbeit mit dem Fachverband Deutsch als Fremdsprache. Bonn: DAAD, 1994 (2. Auflage 1996).

Beier, Rudolf; Möhn, Dieter: »Fachsprachlicher Deutschunterricht. Vorüberlegungen $\mathrm{zu}$ einem >Hamburger Gutachten über fachsprachliche Lehr- und Lernmaterialien des Deutschen als Fremdsprache«, Jahrbuch Deutsch als Fremdsprache 8 (1982), 287-321.

Das Zertifikat Deutsch als Fremdsprache. Hrsg. vom Deutschen Volkshochschulbund und Goethe-Institut. Frankfurt a. M. 1977.

Deutsch als Fremdsprache. Zeitschrift für Theorie und Praxis des Deutschunterrichts für Ausländer. Hrsg. vom Herder-Institut Leipzig. Seit 1991 München: Langenscheidt.

Deutsch als Fremdsprache. Die Deutsche Sprachprüfung für den Hochschulzugang (DSH). Hrsg. vom Deutschen Akademischen Austauschdienst in Zusammenarbeit mit dem Fachverband Deutsch als Fremdsprache. Bonn: DAAD, 1997.

Dresdner Erklärung des Fachverbandes Deutsch als Fremdsprache zur Zukunft des Deutschen als Fremdsprache. Fachverband Deutsch als Fremdsprache, fadaf dokumente 1, Münster 1995 (zu erhalten bei der Geschäftsstelle des FaDaF).

Dietrich, Rainer: »Ein Studium und einige Fragen «, Jahrbuch Deutsch als Fremdsprache 1 (1975), 142-148.

Dietrich, Rainer; Heuer, Fritz: "Deutsch als Fremdsprachenphilologie. Das Heidelberg Modell«, Die Unterrichtspraxis 2 (1975), 54-62.

Eggers, Dietrich: »Zur Neukonzeption des Sprachlehrangebots Deutsch als Fremdsprache an den Hochschulen auf Grund veränderter Anforderungsprofile«, Germanistische Linguistik (erscheint 1997).

Ehnert, Rolf (Hrsg.): Einführung in das Studium des Fachs Deutsch als Fremdsprache. Handreichungen für den Studienbeginn (Werkstattreihe Deutsch als Fremdsprache 1). 2. Auflage Frankfurt a. M.: Lang, 1989.

Ehnert, Rolf (Zusammenstellung): »Studiengänge Deutsch als Fremdsprache in der Bundesrepublik Deutschland. Ein Studienführer«. Hrsg. vom Deutschen Akademischen Austauschdienst. Bonn: DAAD, 1988. 
Fremdsprache Deutsch. Zeitschrift für die Praxis des Deutschunterrichts. Hrsg. vom Vorstand des Goethe-Instituts und Peter Bimmel, Hans-Jürgen Krumm, Gerhard Neuner. München: Klett Edition Deutsch.

Für Sie gelesen: Kommentare und Rezensionen zu über hundert Neuerscheinungen für das Fach Deutsch als Fremdsprache (Nr. 2/3 eines jeden Jahrgangs von Info DaF).

Glück, Helmut; Schmöe, Friederike: Vademecum Deutsch als Fremdsprache (Dr. Rabes Hochschulschriften 1). Bamberg 1995.

Handbuch Deutsch als Fremdsprache (hrsg. von Heinrich Aust, Lutz Götze, Gerhard Helbig u. a.): Berlin; New York: de Gruyter (in Arbeit).

Henrici, Gert: Studienbuch: Grundlagen für den Unterricht im Fach Deutsch als Fremd- und Zweitsprache (und anderer Fremdsprachen) (Studienbücher zu Sprach und Literaturdidaktik 4). Paderborn: Schöningh, 1986.

Henrici, Gert; Riemer, Claudia (Hrsg.): Einführung in die Didaktik des Unterrichts Deutsch als Fremdsprache mit Videobeispielen. 2 Bde. Baltmannsweiler: Schneider Hohengehren, 1994.

Henrici, Gert; Koreik, Uwe (Hrsg.): Deutsch als Fremdsprache. Wo warst Du, wo bist $D u$, wohin gehst Du? Baltmannsweiler: Schneider Hohengehren, 1994.

Heyd, Gertraude: Deutsch lehren. Grundwissen für den Unterricht in Deutsch als Fremdsprache. 2. Auflage Frankfurt a. M.: Diesterweg, 1991.

»Hochschulen und Hochschulpolitik vor neuen Herausforderungen». Beschluß der Kultusministerkonferenz vom 28.2.1997. Dokumentation Nr. 3/1997, 14. März 1997. Hrsg von der Konferenz der Rektoren und Präsidenten der Hochschulen in der Bundesrepublik Deutschland.

Huneke, Hans-Werner; Steinig, Wolfgang: Deutsch als Fremdsprache. Eine Einführung. Berlin; Bielefeld; München: Erich Schmidt, 1997.

Ickler, Theodor: Deutsch als Fremdsprache. Eine Einführung in das Studium. Tübingen: Niemeyer, 1984.

Informationen Deutsch als Fremdsprache (Info DaF). Hrsg. vom Deutschen Akademischen Austauschdienst in Zusammenarbeit mit dem Fachverband Deutsch als Fremdsprache. München: iudicium, 1974ff. Redaktionsadresse: Deutscher
Akademischer Austauschdienst, Referat 201, Kennedyallee 50, D-53175 Bonn.

Jahrbuch Deutsch als Fremdsprache. Hrsg. von Alois Wierlacher und Dietrich Eggers, Konrad Ehlich (seit 1997), Ulrich Engel, Andreas F. Kelletat (seit 1995), Hans-Jürgen Krumm, Dietrich Krusche, Robert Picht (bis 1994), Kurt-Friedrich Bohrer (Dokumentation). München: iudicium, $1975 \mathrm{ff}$.

Kast, Bernd; Neuner, Gerhard (Hrsg.): Zur Analyse, Begutachtung und Entwicklung von Lehrwerken für den fremdsprachlichen Deutschunterricht (Fremdsprachenunterricht in Theorie und Praxis). Berlin; München: Langenscheidt, 1994.

Kleppin, Karin: »Lehrwerkkritik, Lehrwerkanalyse, Lehrwerkforschung. Ein Versuch $\mathrm{zu}$ einer empirisch begründeten Forschungseinrichtung ", Info DaF 1984, 16-23.

Lehrwerkforschung - Lehrwerkkritik. Deutsch als Fremdsprache. Hrsg. von Hans-Jürgen Krumm. München: Goethe-Institut, 1982.

Mannheimer Gutachten zu ausgewählten Lehrwerken Deutsch als Fremdsprache. (Im Auftrag des Auswärtigen Amtes der Bundesrepublik Deutschland). Band 1. Vorgelegt von Ulrich Engel, Wolfgang Halm, HansJürgen Krumm u. a., Heidelberg (4) 1977, Band 2. Erstellt von Ulrich Engel, HansJürgen Krumm, Alois Wierlacher unter Mitarbeit von Wolf-Dietrich Ortmann. Heidelberg 1979.

Materialien Deutsch als Fremdsprache (Mat$D a F)$, hrsg. vom Fachverband Deutsch als Fremdsprache. Redaktionsadresse: Dr. Armin Wolff, Universität Regensburg, Lehrgebiet Deutsch als Fremdsprache, Universitätsstr. 31, D-93053 Regensburg. (Ein Übersicht über die in dieser Reihe erschienenen Titel ist als Anlage 3 diesem Beitrag hinzugefügt.)

Nodari, Claudio: Perspektiven einer neuen Lehrwerkkultur. Aarau: Sauerländer, 1995.

Rahmenordnung für ausländische Studienbewerber, den Unterricht an den Studienkollegs und die Feststellungsprüfung. Beschluß der KMK vom 15.4.1994.

»Rahmenordnung für die Prüfung zum Nachweis deutscher Sprachkenntnisse (PNdS) für ausländische Studienbewerber an den Wissenschaftlichen Hochschulen der Bundesrepublik Deutschland einschließlich Berlin (West) (Beschluß der 101. WRK vom 12.12.1972). Abgedruckt in: »Tests und Prüfungen 1974/75 aus 
den Lehrgebieten Deutsch als Fremdsprache an den Hochschulen und Universitäten«, MatDaF, Heft 5, Mainz 1975, 137-141, und in: Jahrbuch Deutsch als Fremdsprache 1 (1975), 153-155.

»Rahmenordnung für die Prüfung zum Nachweis deutscher Sprachkenntnisse (PNdS) für ausländische Studienbewerber an den wissenschaftlichen Hochschulen der Bundesrepublik Deutschland einschließlich Berlin (West) « (Beschluß des 140. Plenums RK vom 4./5. Juli 1983 unter Zustimmung der Kultusministerkonferenz vom 27. Juni 1983). In: Deutsch als Fremdsprache an den Hochschulen und Studienkollegs der Bundesrepublik Deutschland einschließlich Berlin (West): Die Sprachlehrangebote. Zusammengestellt von Armin Wolff, Gabriele Neuf-Münkel und Dietrich Eggers, hrsg. vom Deutschen Akademischen Austauschdienst. 3. Aufl. Bonn: DAAD, 1988, 177-181.

»Rahmenordnung für die Deutsche Sprachprüfung für den Hochschulzugang ausländischer Studienbewerber (DSH) « (Beschluß des 172. Plenums (21./22.2.1994) und des 72. Senats (30.5.1995) der Hochschulrektorenkonferenz (HRK), Info DaF 23 (1996), 110-116. Außerdem in: Deutsch als Fremdsprache an den Hochschulen und Studienkollegs in Deutschland: Die Sprachlehrangebote. Zusammengestellt von Armin Wolff, hrsg. vom Deutschen Akademischen Austauschdienst. 4. Aufl. Bonn: DAAD, 1996, 185-190. (Alle im Jahr 1981 geltenden Rahmenordnungen, Rahmenpläne und Prüfungsordnungen wurden in einer Zusammenstellung von Rolf Ehnert und Gabriele Neuf-Münkel abgedruckt in: Info DaF 6 (1981), 1-158).

»Rahmenplan Deutsch als Fremdsprache für `Sprachlehrveranstaltungen vor Aufnahme des Fachstudiums « und für studienbegleitende Lehrveranstaltungen an den Universitäten und Hochschulen der Bundesrepublik Deutschland ", Info DaF 1979, H. 2, 1-41.

Rösler, Dietmar: Deutsch als Fremdsprache. Stuttgart; Weimar: Metzler, 1994.

Special Language - Fachsprache. Internationale Zeitschrift für Fachsprachenforschung, Didaktik und Terminologie. Wien: Braunmüller.

Strauß, Dieter: Didaktik und Methodik Deutsch als Fremdsprache. Eine Einführung.
Berlin; München; Wien; Zürich; New York: Langenscheidt, 1984.

»Strukturmodell Lehr- und Forschungsgebiet >Deutsch als Fremdsprache«", Info DaF 1974, H. 2, 11-12.

»Struktur- und Organisationsmodell für die Ausbildung ausländischer Studenten in der deutschen Sprache (>Lehrgebiet Deutsch als Fremdsprache «)«, Info DaF 1975, H. 2, 8-13.

Studien- und Wissenschaftsstandort Deutschland. Aktionsprogramm des DAAD zur Förderung des Studiums von Ausländern an deutschen Hochschulen. Bonn: DAAD, Januar 1997.

Studienvorbereitung an den Studienkollegs für die Fachhochschulen. Hinweise für ausländische Studienbewerber. Hrsg. vom Deutschen Akademischen Austauschdienst Bonn: DAAD 1995.

Schulte, Hansgert: "Einführung zum Start der >Informationen Deutsch als Fremdsprache" ", Info DaF 1974, H. 1, 3.

Schulte, Hansgert: "Deutsch als Fremdsprache und die Internationalität der Hochschulen: 15 Jahre Arbeitskreis Deutsch als Fremdsprache beim DAAD «, MatDaF Heft 29, für den FaDaF hrsg. von Armin Wolff und Helmut Rössler. Regensburg 1990.

»Verfahrensordnung für die Jahrestagung und den Arbeitskreis Deutsch als Fremdsprache", Info DaF 1974, H. 1, 4-6.

Universitätsgesetz des Landes Rheinland-Pfalz. Ministerium für Bildung, Wissenschaft und Weiterbildung (Hrsg).

Weinrich, Harald: »Deutsch als Fremdsprache - Konturen eines neuen Faches", MatDaF Heft 14, für den AKDaF hrsg. von Armin Wolff. Regensburg 1979.

Welter Winfried; Moll, Birgitta: FaDaF Aktuell« Mitgliederzeitschrift des Fachverbandes Deutsch als Fremdsprache (FaDaF). Münster: FaDaF-Geschäftsstelle, $1995 \mathrm{ff}$.

Wierlacher, Alois: "Sprachunterricht und Kulturvermittlung. Prolegomena zu einer Kritik deutscher Sprachlehren für Ausländer", Info DaF, Sondernummer. Bonn: DAAD 1974, 4-9.

Wierlacher, Alois: „Germanistik und Ausländerstudium «. In: Werner, Otmar; Fritz, Gert (Hrsg.), Deutsch als Fremdsprache und neuere Linguistik. München: Hueber 1975, 289-297.

Wierlacher, Alois: »Überlegungen zur Begründung eines Ausbildungsfaches 
>Deutsch als Fremdspracher «, Jahrbuch Deutsch als Fremdsprache 1 (1975), 119-136.

Wierlacher, Alois (Hrsg.): Fremdsprache Deutsch. Grundlagen und Verfahren der Germanistik als Fremdsprachenphilologie. München: Fink, 1980 (Band 1) und 1981 (Band 2).

Wittmann, Lothar: „Ein zentrales politisches Anliegen. Förderung der deutschen Sprache im Ausland ", Forschung und Lehre 2 (1996), 69-72.

Wolff, Armin; Neuf-Münkel, Gabriele; Eggers, Dietrich (Zusammenstellung): Deutsch als Fremdsprache an den Hochschulen und Studienkollegs der Bundesrepublik Deutschland einschließlich Berlin (West): Die Sprachlehrangebote. 1. Aufl. 1978; 2. Aufl. 1982; 3. Aufl. 1988. Hrsg. vom Deutschen Akademischen Austauschdienst. Bonn: DAAD.

Wolff, Armin: »Leitlinien der Lehrerausbildung im Bereich Deutsch als Fremdsprache $«$, MatDaF, Heft 19, für den AKDaF hrsg. von Armin Wolff, Aloys Beuers und Rolf Ehnert: Regensburg 1982, 67-76.

Wolff, Armin; Hoffmann, Joachim; Rug, Wolfgang: "Einführung zur ersten Ausgabe von Info DaF", Info DaF 1 (1974), 2.

Wolff, Armin (Zusammenstellung): Deutsch als Fremdsprache an den Hochschulen und Studienkollegs in Deutschland: Die Sprachlehrangebote. Hrsg. vom Deutschen Akademischen Austauschdienst. 4. Aufl. Bonn: DAAD, 1996.

Wolff, Armin: "Die neue Rahmenordnung für die >Deutsche Sprachprüfung für den Hochschulzugang ausländischer Studienbewerber (DSH) «, Info DaF 23 (1996), 109-116.

Zielsprache Deutsch. Zeitschrift für Unterrichtsmethodik und angewandte Sprachwissenschaft. Ismaning: Hueber, 1970ff.

Zugang zum Fachstudium an den wissenschaftlichen Hochschulen in der Bundesrepublik Deutschland über die Studienkollegs für ausländische Studierende. Hinweise für ausländische Studienbewerber. Hrsg. vom Deutschen Akademischen Austauschdienst Bonn: DAAD 1995.
Anlage 1: Jahrestagungen des Arbeitskreises / Fachverbandes Deutsch als Fremdsprache

mit den folgenden Themenschwerpunkten:

1973 in Erlangen: »Didaktische und methodische Beiträge Deutsch als Fremdsprache". In: Materialien Deutsch als Fremdsprache, Heft 1, für den $\mathrm{AKDaF}$ hrsg. von Dietrich Eggers, Mainz 1975, 185 Seiten.

1974 in Trier: »Didaktische und methodische Beiträge Deutsch als Fremdsprache«. In: Materialien Deutsch als Fremdsprache, Heft 4, für den $\mathrm{AKDaF}$ hrsg. von Dietrich Eggers, Mainz 1976, 126 Seiten.

1975 in Berlin: »Lernzielformulierung im Bereich Deutsch als Fremdsprache" »Deutsch für ausländische Arbeitnehmer und Kinder ausländischer Arbeitnehmer in der Bundesrepublik Deutschland«. In: Materialien Deutsch als Fremdsprache, Heft 7, für den $\mathrm{AKDaF}$ hrsg. von Dietrich Eggers, Mainz 1977, 143 Seiten.

1976 in Bremen: »Kontrastivität, Fehleranalyse, Unterrichtspraxis Deutsch als Fremdsprache". In: Materialien Deutsch als Fremdsprache, Heft 9, für den AKDaF hrsg. von Wolfgang Rug und Kurt Werner Jauß, Regensburg 1978, 304 Seiten.

1977 in Mainz: "Studienbegleitende Lehrveranstaltungen Deutsch als Fremdsprache" - »Mündliche Leistungen und deren Beurteilung«. In: Materialien Deutsch als Fremdsprache, Heft 11, für den $\mathrm{AKDaF}$ hrsg. von Armin Wolff und Dietrich Eggers, Regensburg 1978, 310 Seiten.

1978 in Bonn: »Forschungsergebnisse sogenannter Nachbarwissenschaften (wie Lernpsychologie, Linguistik, Psycholinguistik) und ihre Applizierbarkeit auf die Bereiche des Deutschen als Fremdsprache " - Grundsatzdiskussion über studienbegleitende Lehrveranstaltungen Deutsch als Fremdsprache" »Erfahrungsaustausch über Ansätze zu Stu- 
diengängen/Teilstudiengängen Deutsch als Fremdsprache". In: Materialien Deutsch als Fremdsprache, Heft 14, für den AKDaF hrsg. von Armin Wolff und Kurt Werner Jauß, Regensburg 1979, 309 Seiten.

1979 in Regensburg: »Diskussion des Rahmenplans Deutsch als Fremdsprache an den Hochschulen" - "Ergebnisse empirischer Fremdsprachenerwerbsforschung im Bereich Deutsch als Fremdsprache - »Literaturvermittlung des Faches Deutsch als Fremdsprache" - "Zur Ausbildung von DaF-Lehrern für Kinder ausländischer Arbeitnehmer «. In: Materialien Deutsch als Fremdsprache, Heft 15, für den AKDaF hrsg. von Armin Wolff, Regensburg 1980, 224 Seiten.

1980 in Bielefeld: »Analyse und Evaluation von Lehrmaterialien für Deutsch als Fremdsprache im In- und Ausland «. In: Materialien Deutsch als Fremdsprache, Heft 18, für den AKDaF hrsg. von Armin Wolff, Rolf Ehnert und Werner Jauß, Regensburg 1981, 299 Seiten, ISBN 3-88246-044$\mathrm{X}$.

1981 in Münster: »Die Aus- und Weiterbildung von Lehrern für Deutsch als Fremdsprache" In: Materialien Deutsch als Fremdsprache, Heft 19, für den AKDaF hrsg. von Armin Wolff, Aloys Beuers und Rolf Ehnert, Regensburg 1982, 301 Seiten, ISBN 3-88246-057-1.

1982 in Aachen: »Schwierigkeiten ausländischer Studierender in der Anfangsphase des Fachstudiums und Möglichkeiten studienbegleitender Lehrveranstaltungen " - »Vorkurse (Sozialisationskurse) für ausländische Studienanfänger" - »Literatur ausländischer Arbeitnehmer in der Bundesrepublik Deutschland (»Gastarbeiterliteratur «)«. In: Materialien Deutsch als Fremdsprache, Heft 22, für den AKDaF hrsg. von Armin Wolff, Erhard Heilmann und Haluk Turat, Regensburg 1983, 414 Seiten, ISBN 388246-077-6.
1983 in Bayreuth: »Deutsch als Fremdsprache und Germanistik im Aus- und Inland «"Studienprobleme ausländischer Studierender im Fachstudium « - »Aufgaben der Phonetik im Sprachunterricht Deutsch als Fremdsprache" - "Offene Arbeits- und Übungsformen, Übungstypologien und Planung von Unterrichtssequenzen «. In: Materialien Deutsch als Fremdsprache, Heft 23, für den $\mathrm{AKDaF}$ hrsg. von Armin Wolff, und Markus Motsch, Regensburg 1984, 423 Seiten, ISBN 3-88246-088-1.

1984 in Stuttgart: Lexik im Fremdsprachenerwerb " - "Sprachlehrveranstaltungen für Postgraduierte - »Sprachlernspiele - »Zur Didaktik des Faches Deutsch als Fremdsprache . In: Materialien Deutsch als Fremdsprache, Heft 24, für den $\mathrm{AKDaF}$ hrsg. von Armin Wolff, und Wolfgang Hornung, Regensburg 1985, 283 Seiten, ISBN 3-88246-105-5.

1985 in Saarbrücken: »Interkulturelles Lernen am Beispiel von Korea und dem frankophonen Afrika" - »Bildungsziel Zweisprachigkeit? Zur Erziehung ausländischer Kinder in der Bundesrepublik Deutschland " "Forum Deutsch als Fremdsprache «. In: Materialien Deutsch als Fremdsprache, Heft 25, für den $\mathrm{AKDaF}$ hrsg. von Armin Wolff, und Rainer Kohl, Regensburg 1986, 516 Seiten, ISBN 3-88246-110-1.

1986 in Tübingen: »Vermittlung fremder Kultur in Theorie, Didaktik und Praxis" "Neue Lehr- und Lernmethoden und ihre Umsetzung für den DaF-Unterricht « - „Forum Deutsch als Fremdsprache". In: Materialien Deutsch als Fremdsprache, Heft 27, für den AKDaF hrsg. von Armin Wolff und Wolfgang Rug, Regensburg 1987, ix, 309 Seiten, ISBN 3-88246-132-2.

1987 in Karlsruhe: "Rahmenbedingungen des Ausländerstudiums - Didaktik der Wissenschaftspropädeutik und Sprachvermittlung" - "Leseverstehen, Textaufgaben, Schreiben « - „Forum Deutsch als Fremd- 
sprache«. In: Didaktik der Wissenschaftspropädeutik und Sprachvermittlung (= Materialien Deutsch als Fremdsprache, Heft 31), für den AKDaF hrsg. von Armin Wolff, Klaus Dieter Justen und Harald Klingel, Regensburg 1991, 350 Seiten, ISBN 3-88246-159-4.

1988 in Kiel: »Heterogene Lernergruppen «Arbeitstechniken der Lehrenden; Deutsche DaF-Lehrende im Ausland « - »Forum Deutsch als Fremdsprache « In: Materialien Deutsch als Fremdsprache, Heft 30, für den AKDaF hrsg. von Armin Wolff und Horst Zindler, Regensburg 1991, V, 424 Seiten, ISBN 3-88246-158-6.

1989 in Freiburg: »Deutsch als Fremdsprache in Europa - »Grammatik« - "Landeskunde" - »Literatur « - „Empirische Aspekte im Bereich Deutsch als Fremdsprache" "Arbeitstechniken « - „Forum Deutsch als Fremdsprache«. In: Materialien Deutsch als Fremdsprache, Heft 29, für den AKDaF hrsg. von Armin Wolff und Helmut Rössler, Regensburg 1990, VII, 440 Seiten, ISBN 3-88246-150-0.

1990 in Bonn: »Deutsch als Fremdsprache im europäischen Binnenmarkt « - Grammatik und Grammatikvermittlung « - Fiktionale Texte im Sprachunterricht " - Computer und Computerunterstützung im Bereich Deutsch als Fremdsprache" - »Forum Deutsch als Fremdsprache«. In: Deutsch als Fremdsprache im europäischen Binnenmarkt (= Materialien Deutsch als Fremdsprache, Heft 33), für den $\mathrm{FaDaF}$ hrsg. von Armin Wolff, Regensburg 1993, IX, 395 Seiten, ISBN 3-88246-161-6.

1991 in Berlin: »Prüfen und Testen " »Wortschatzarbeit « - „Landeskunde neu« »Deutsch als Fremdsprache im Ausland « "Forum Deutsch als Fremdsprache«. In: Materialien Deutsch als Fremdsprache, Heft 35, für den FaDaF hrsg. von Armin Wolff und Barbara Gügold, Regensburg 1994, VII, 299 Seiten, ISBN 3-88246-165-9.
1992 in Münster: »Mündliche Kommunikation" - »Unterrichts- und Übungsformen DaF" - »Themen- und zielgruppenspezifische Auswahl von Unterrichtsmaterialien «"Modelle für studien- und berufsbegleitenden Unterricht " - "Forum Deutsch als Fremdsprache«. In: Materialien Deutsch als Fremdsprache, Heft 40, für den $\mathrm{FaDaF}$ hrsg. von Armin Wolff und Winfried Welter, Regensburg 1995, VI, 384 Seiten, ISBN 3-88246-180-2.

1993 in Erlangen: »Autonomes Lernen «"Lernpsychologie im Fremdsprachenunterricht «-»Deutsch als Fremdsprache im internationalen Kontakt « - "Qualitätskriterien für Sprachkurse DaF im außeruniversitären Bereich « - »Forum Deutsch als Fremdsprache«. In: Materialien Deutsch als Fremdsprache, Heft 42, für den FaDaF hrsg. von Armin Wolff, Anette Köppel und Anneliese Stein-Meintker, Regensburg 1996, V, 221 Seiten, ISBN 3-88246-183-7.

1994 in Aachen: »Fach- und Sprachunterricht. Gemeinsamkeiten und Unterschiede" »Studienkollegs Deutsch als Fremdsprache. Von der Theorie zur Praxis" - "Forum Deutsch als Fremdsprache «. In: Materialien Deutsch als Fremdsprache, Heft 43, für den FaDaF hrsg. von Armin Wolff und Walter Schleyer, Regensburg 1996, 480 Seiten, ISBN 3-88246-186-1.

1995 in Dresden: »Ausländerstudium in einem sich verändernden Deutschland « - Multimedia und Fremdsprachenlernen « »Üben und Prïfen in Fach- und Berufssprachen " "Landeskunde, kulturelle Kompetenz - - Forum Deutsch als Fremdsprache« (= DaF für die Zukunft. Eine Zukunft für DaF!). In: Materialien Deutsch als Fremdsprache, Heft 44, für den FaDaF hrsg. von Armin Wolff und Dagmar Blei, Regensburg 1997, 480 Seiten, ISBN 3-88246-187-X.

1996 in Göttingen: »Gehirn, Gedächtnis, Fremdsprachenlernen « - »Prozeßorientiertes Fremdsprachenlernen " - »Deutschlehreraus- 
bildung in West- und Osteuropa - »Eine deutsche Literatur, AutorInnen nichtdeutscher Muttersprache". In: Materialien Deutsch als Fremdsprache, Heft 46, für den FaDaF hrsg. von Armin Wolff, Gisela Tütken und Horst Liedtke, Regensburg 1997, VII, 440 Seiten, ISBN 3-88246-190-X.

1997 in Mainz: »Emotion und Kognition beim Fremdsprachenlernen " - Lernen mit neuen Medien " - „Deutsch als Fremdsprache und die Attraktivität des Studien- und Lernortes Deutschland " - Forum Deutsch als Fremdsprache«. In: Materialien Deutsch als Fremdsprache, Heft 47, für den FaDaF hrsg. von Armin Wolff und Dietrich Eggers, Regensburg 1998 (in Vorbereitung).

Anlage 2: Fachtagungen des Arbeitskreises / Fachverbandes Deutsch als Fremdsprache

Der Arbeitskreis/Fachverband Deutsch als Fremdsprache hat (teilweise in Zusammenarbeit mit anderen Organisationen) folgende Fachtagungen durchgeführt:

1975 in Ludwigsburg: »Deutschlandkunde, Kulturwissenschaft und Sprachdidaktik, Teil I (in Zusammenarbeit mit dem DeutschFranzösischen Jugendwerk).

1975 in Heidelberg: "Linguistische und psychologische Bedingungen des Fremdsprachenerwerbs" (in Zusammenarbeit mit dem Goethe-Institut und mit der Deutschen Abteilung der Fachgruppe Angewandte Linguistik der Universität Heidelberg).

1976 in Ludwigsburg: »Deutschlandkunde, Kulturwissenschaft und Sprachdidaktik, Teil $I I \ll$ (in Zusammenarbeit mit dem Deutsch-Französischen Jugendwerk).

1977 in Freiburg: »Probleme des Deutschunterrichts für Lerner mit außereuropäischen Ausgangssprachen in heterogenen Gruppen«.
1979 in Königstein: »Landeskunde der Herkunftsländer«.

1980 in München: »Adressatenspezifischer Einsatz von Videoaufzeichnungen in LehrLern- und Informationsveranstaltungen $D a F \ll$ (1. Internationale Fachtagung Video, in Zusammenarbeit mit dem Goethe-Institut).

1981 in Göttingen: »Prüfung zum Nachweis deutscher Sprachkenntnisse für ausländische Studierende«.

1981 in München: »Video im Fremdsprachenunterricht « (2. Internationale Fachtagung Video, in Zusammenarbeit mit dem Goethe-Institut).

1983 in Radolfzell: »Didaktisierung von Fachtexten im Sprachunterricht Deutsch als Fremdsprache".

1983 in Mainz: »Sprachlernspiele«.

1984 in München: »3. Internationale VideoTagung " (in Zusammenarbeit mit dem Goethe-Institut).

1984 in Aachen: »Deutsche Literatur in einem fremden Land"

1985 in München: Workshop im Rahmen der GAL: »Fachsprachen und ihre Vermittlung im Bereich DaF«.

1986 in Germersheim: »Übersetzungswissenschaften und Deutsch als Fremdsprache".

1986 in Wieseneck: "Didaktik des Faches Deutsch als Fremdsprache - Teil I: Praxis und Theorieansätze zu Hörverstehen und Leseverstehen «.

1986 in Mainz: Workshop im Rahmen der GAL: »Psycholinguistische Aspekte des Lernens im Bereich DaF/DaZ«.

1987 in Göttingen: Fachtagung zur PNdS: "Prüfung zum Nachweis deutscher Sprachkenntnisse für ausländische Studienbewerber «. 
1987 in Mainz: »Didaktik des Faches Deutsch als Fremdsprache - Teil II: Grammatik«.

1987 in Heidelberg: Workshop im Rahmen der GAL: "Deutsch als Fremdspracheein Wirtschaftsfaktor «.

1988 in Regensburg: »Didaktik des Faches Deutsch als Fremdsprache III: Landeskunde «.

1988 in Trier: Workshop im Rahmen der GAL: Landeskunde des deutschsprachigen Raumes".

1989 in Mainz: »Studienbegleitende Lehrveranstaltungen «.

1989 in Göttingen: Workshop im Rahmen der GAL: »Interkulturelle Kommunikation im Bereich Deutsch als Fremdsprache".

1990 in Regensburg: »Phonetik Ausspracheschulung, Sprecherziehung im Bereich Deutsch als Fremdsprache" (in Zusammenarbeit mit der Deutschen Gesellschaft für Sprecherziehung und Sprechwissenschaft).

1990 in Bonn: Workshop im Rahmen der GAL: »Deutsch als Fremdsprache in den neunziger Jahren «.

1991 in Göttingen: »Schreiben «.

1991 in Leipzig: „Studienbegleitende Deutschkurse«.

1991 in Mainz: Workshop im Rahmen der GAL: "Zu einigen Perspektiven des Faches DaF für die neunziger Jahre«.

1992 in Regensburg: »Reform der PNdSPrüfung «.

1992 in Chemnitz: »Deutschsprachige Fachkommunikation im universitären und außeruniversitären Bereich «.

1993 in Leipzig: »DaF-Unterricht im Fachunterricht«.

1995 in Jena: »Medieneinsatz im Fremdsprachenunterricht unter besonderer Berücksichtigung von Multimedia«.
1996 in Regensburg: Fachtagung zur DSH I: »Umsetzung der neuen DSH-Ordnung in die Prüfungspraxis".

1997 in Regensburg: Fachtagung zur DSH II: »Umsetzung der neuen DSH-Ordnung in die Prüfungspraxis«.

\section{Anlage 3: Die Reihe Materialien Deutsch als Fremdsprache (MatDaF)}

Bestellungen an:

Dr. Armin Wolff, Universität Regensburg, Lehrgebiet Deutsch als Fremdsprache Universitätsstraße 31, D-93053 Regensburg, Tel.: (0941) 943-2426, Tel. (0941) 943-2425 oder (0941) 943-3008, Fax: (0941) 943-2410, e-mail: Armin.Wolff@ sprachlit.uni-regensburg.de.

\section{Heft 1}

Eggers, Dietrich (Hrsg.): Didaktische und methodische Beiträge Deutsch als Fremdsprache. Arbeitsmaterialien der 1. Jahrestagung DaF 1973 in Erlangen. 1. Auflage Mainz 1975, broschiert DM 12,00, 185 Seiten (vergriffen).

\section{Heft 2}

Rug, Wolfgang: Deutsch als FremdspracheEine Aufgabe für die Lehrerfortbildung. Ein Nachbericht zum Fortbildungskurs Deutsch als Fremdsprache in Mannheim vom 26.2. bis 9.3.1975. 1. Auflage Mainz 1975, 3. Auflage 1998, broschiert DM 3,00, 42 Seiten (vergriffen).

\section{Heft 3}

Rug, Wolfgang (Hrsg.): Berichte aus den Lehrgebieten. Teil 1: Heidelberg, Hamburg, FU Berlin. 1. Auflage Mainz 1975, broschiert DM 6,00, 67 Seiten (vergriffen)

Heft 4

Rug, Wolfgang (Hrsg.): Didaktische und methodische Beiträge Deutsch als Fremdsprache. Arbeitsmaterialien der 2. Jahrestagung $\mathrm{DaF}$ 
1974 in Trier. 1. Auflage Mainz 1975, broschiert DM 10,00, 126 Seiten (vergriffen).

\section{Heft 5}

Stein, Anneliese; Wiegand-Kanzaki, Anneliese; Wolff, Armin (Hrsg.): Tests und Prüfungen 1974/75 aus den Lehrgebieten Deutsch als Fremdsprache an den Hochschulen und Universitäten. 1. Auflage Mainz 1975, broschiert DM 8,80, 143 Seiten, ISBN 3-88246-050-4 (vergriffen).

\section{Heft 6}

Stein, Anneliese; Neuf-Münkel, Gabriele; Wolff, Armin (Hrsg.): Feststellungsprüfungen 1974/75 aus den Studienkollegs der Hochschulen und Universitäten. 1. Auflage Mainz 1976, broschiert DM 7,00, 89 Seiten (vergriffen).

\section{Heft 7}

Dietrich, Rainer; Wolff, Armin (Hrsg.): Didaktische und methodische Beiträge Deutsch als Fremdsprache. Arbeitsmaterialien der 3. Jahrestagung DaF 1975 in Berlin. 1. Auflage Mainz 1976, broschiert DM 11,50, 143 Seiten (vergriffen).

\section{Heft 9}

Rug, Wolfgang; Jauß, Kurt Werner: (Hrsg.): Kontrastivität - Fehleranalyse Unterrichtspraxis Deutsch als Fremdsprache. Beiträge und Materialien der 4. Jahrestagung DaF 1976 in Bremen. 1. Auflage Regensburg 1978, broschiert DM 10,80, 304 Seiten (vergriffen).

\section{Heft 10}

Wagner, Johannes: Spielübungen und Übungsspiele im Fremdsprachenunterricht. 1. Auflage Regensburg 1977; 6. Auflage 1987, broschiert DM 9,00, 107 Seiten, ISBN 3-88246-049-0 (vergriffen).

\section{Heft 11}

Eggers, Dietrich (Hrsg.): Studienbegleitende Lehrveranstaltungen Deutsch als Fremdsprache - Mündliche Leistungen und deren Beurteilung. Beiträge der 5. Jahrestagung DaF 1977 in Mainz. 1. Auflage Regens- burg 1978, broschiert DM 13,20, 310 Seiten (vergriffen).

\section{Heft 14}

Wolff, Armin; Jauß, Kurt Werner (Hrsg.): Forschungsergebnisse sogenannter Nachbarwissenschaften (wie Lernpsychologie, Linguistik, Psycholinguistik) und ihre Applizierbarkeit auf die Bereiche des Deutschen als Fremdsprache - Grundsatzdiskussion über studienbegleitende Lehrveranstaltungen Deutsch als Fremdsprache - Erfahrungsaustausch über Ansätze zu Studiengängen/Teilstudiengängen Deutsch als Fremdsprache. Beiträge der 6. Jahrestagung DaF 1978 in Bonn. 1. Auflage Regensburg 1979, broschiert, DM 13,50/ 10,00*, 309 Seiten (vergriffen).

\section{Heft 15}

Wolff, Armin; Dietrich, Rainer; Ehnert, Rolf (Hrsg.): Diskussion des Rahmenplans Deutsch als Fremdsprache an den Hochschulen - Ergebnisse empirischer Fremdsprachenerwerbsforschung im Bereich Deutsch als Fremdsprache - Literaturvermittlung des $\mathrm{Fa}$ ches Deutsch als Fremdsprache - Zur Ausbildung von DaF-Lehrern für Kinder ausländischer Arbeitnehmer. Beiträge der 7. Jahrestagung DaF 1979 in Regensburg. 1. Auflage Regensburg 1980, broschiert DM 12,50, 224 Seiten (vergriffen).

\section{Heft 16}

Vorderwülbecke, Klaus; Wintermann, Bernd (Hrsg.): Vom Sprachunterricht zum Kulturunterricht. Dokumentation der Fachtagung des AKDaF 1979: >Landeskunde der Herkunftsländer . 1. Auflage Regensburg 1980, broschiert DM 13,50, 265 Seiten, ISBN 3-88246-041-5 (vergriffen)

\section{Heft 17}

Arbeitsgruppe "Linguistik " des DAAD in Frankreich: Verbwörterbücher und Verbvalenz im Deutschunterricht für Ausländer. 1. Auflage Regensburg 1980, broschiert DM 8,50, 119 Seiten, ISBN 3-88246046-6 (vergriffen). 
Heft 18

Wolff, Armin; Ehnert, Rolf; Jauß, Kurt Werner (Hrsg.): Analyse und Evaluation von Lehrmaterialien für Deutsch als Fremdsprache im In- und Ausland. Beiträge der 8. Jahrestagung DaF 1980 in Bielefeld. 1. Auflage Regensburg 1981, broschiert DM 16,00, 299 Seiten, ISBN 3-88246-044-X (vergriffen).

\section{Heft 19}

Wolff, Armin; Beuers, Aloys; Ehnert, Rolf (Hrsg.): Die Aus- und Weiterbildung von Lehrern für Deutsch als Fremdsprache. Beiträge der 9. Jahrestagung DaF 1981 in Münster. 1. Auflage Regensburg 1982, 301 Seiten, broschiert DM 19,50/16,00*, ISBN 3-88246-057-1.

\section{Heft 20}

Zindler, Horst (Zusammenstellung): Texte zum Hörverstehen und zur Mitschrift. 2. verbesserte Auflage Regensburg 1987, 223 Seiten, broschiert DM 15,00/12,00*, ISBN 3-88246-129-2.

\section{Heft 21}

Großkopf, Sabine: Kulturschock und Fremdverhaltensunterricht. Ausländische Studenten in der BRD. Diss. Hamburg 1982. 1. Auflage Regensburg 1982, 425 Seiten, broschiert DM 21,50/17,00*, ISBN 3-88246-059-8.

\section{Heft 22}

Wolff, Armin; Heilmann, Erhard; Turat, Halek (Hrsg.): Schwierigkeiten ausländischer Studierender in der Anfangsphase des Fachstudiums und Möglichkeiten studienbegleitender Lehrveranstaltungen; Vorkurse (Sozialisationskurse) für ausländische Studienanfänger; Literatur ausländischer Arbeitnehmer in der Bundesrepublik Deutschland (»Gastarbeiterliteratur«). Beiträge der 10. Jahrestagung DaF 1982 in Aachen, 1. Auflage Regensburg 1983, 414 Seiten, broschiert DM 23,50/19,00*, ISBN 3-88246077-6.
Heft 23

Wolff, Armin; Motsch, Markus (Hrsg.): Deutsch als Fremdsprache und Germanistik im Aus- und Inland; Studienprobleme ausländischer Studierender im Fachstudium; Aufgaben der Phonetik im Sprachunterricht Deutsch als Fremdsprache; offene Arbeitsund Übungsformen; Übungstypologien und Planung von Unterrichtssequenzen. Beiträge der 11. Jahrestagung DaF 1983 in Bayreuth. 1. Auflage Regensburg 1984, 423 Seiten, broschiert DM 25,00/20,00*, ISBN 3-88246-088-1.

\section{Heft 24}

Wolff, Armin; Hornung, Wolfgang (Hrsg.): Lexik im Fremdsprachenerwerb. Sprachlehrveranstaltungen für Postgraduierte. Sprachlernspiele. Zur Didaktik des Faches Deutsch als Fremdsprache. Beiträge der 12. Jahrestagung DaF 1984 in Stuttgart. 1. Auflage Regensburg 1985, 283 Seiten, broschiert DM 20,00/ 16,00*, ISBN 3-88246-105-5.

Heft 25

Wolff, Armin; Kohl, Rainer (Hrsg.): Interkulturelles Lernen am Beispiel von Korea und dem frankophonen Afrika. Bildungsziel Zweisprachigkeit? Zur Erziehung ausländischer Kinder in der Bundesrepublik Deutschland. Forum Deutsch als Fremdsprache. Beiträge der 13. Jahrestagung DaF 1985 in Saarbrücken. 1. Auflage Regensburg 1986, 516 Seiten, broschiert DM 34,00/ 26,00*, ISBN 3-88246-110-1.

\section{Heft 26}

Ehnert, Rolf; Schleyer, Walter (Hrsg.): Übersetzen im Fremdsprachenunterricht. Beiträge zur Übersetzungswissenschaft Annäherungen an eine Übersetzungsdidaktik. Dokumentation der Fachtagung des AKDaF 1986. 1. Auflage Regensburg 1987, 210 Seiten, broschiert DM 25,00, ISBN 3-88246-126-8 (vergriffen).

\section{Heft 27}

Wolff Armin; Rug, Wolfgang (Hrsg.): Vermittlung fremder Kultur: Theorie - Didaktik 
- Praxis. Neue Lehr- und Lernmethoden und ihre Umsetzung für den DaF-Unterricht. Beiträge der 14. Jahrestagung DaF 1986 in Tübingen. 1. Auflage Regensburg 1987, IX, 309 Seiten, broschiert DM 25,00/ 20,00*, ISBN 3-88246-132-2.

\section{Heft 28}

Eggers, Dietrich (Hrsg.): Didaktik des Deutschen als Fremdsprache. HörverstehenLeseverstehen - Grammatik. Beiträge der Fachtagung »Didaktik Deutsch als Fremdsprache« 1988. 1. Auflage Regensburg 1989, V, 220 Seiten, broschiert DM 19,00/15,00*, ISBN 3-88246-151-9.

\section{Heft 29}

Wolff, Armin; Rössler, Helmut (Hrsg.): Deutsch als Fremdsprache in Europa. Empirische Aspekte im Bereich Deutsch als Fremdsprache. Arbeitstechniken. Beiträge der 15. Jahrestagung DaF 1987 in Freiburg. 1. Auflage Regensburg 1990, VII, 438 Seiten, broschiert DM 37,00/33,00*, ISBN 382246-150-0 (vergriffen).

\section{Heft 30}

Wolff, Armin; Zindler, Horst (Hrsg.): Heterogene Lernergruppen: Probleme und Chancen; Arbeitstechniken; Deutsche DaF-Lehrende im Ausland. Beiträge der 16. Jahrestagung DaF 1988 in Kiel. 1. Auflage Regensburg 1991, V, 424 Seiten, broschiert DM 33,00/29,00*, ISBN 3-88246-158-6.

\section{Heft 31}

Wolff, Armin; Justen, Klaus-Dieter; Klingel, Harald (Hrsg.): Rahmenbedingungen des Ausländerstudiums; Didaktik der Wissenschaftspropädeutik und Sprachvermittlung; Phonetik- und Ausspracheschulung im DaF-Unterricht; Leseverstehen - Textaufgaben - Schreiben. Beiträge der 17. Jahrestagung DaF 1989 in Karlsruhe. 1. Auflage Regensburg 1991, 350 Seiten, broschiert DM 35,00/31,00*, ISBN 3-88246-159-4.

\section{Heft 32}

Vorderwülbecke, Klaus (Hrsg.): Phonetik,
Ausspracheschulung und Sprecherziehung im Bereich Deutsch als Fremdsprache. Fachtagung Didaktik Deutsch als Fremdsprache IV. 1. Auflage Regensburg 1992, VIII, 182 Seiten, broschiert DM 30,00/27,00*, ISBN 3-88246-160-8.

\section{Heft 33}

Wolff, Armin (Hrsg.): Deutsch als Fremdsprache im europäischen Binnenmarkt. Beiträge der 18. Jahrestagung $\mathrm{DaF} 1990$ in Bonn. 1. Auflage Regensburg 1993, X, 395 Seiten, broschiert DM 43,00/38,00*, ISBN 3-88246-161-6 (vergriffen).

\section{Heft 34}

Ebert, Harald; Hentschel, Uwe (Hrsg.): Ausländerstudium in interkulturellem Kontext. 1. Auflage Regensburg 1991, 285 Seiten, broschiert DM 28,00/25,00*, ISBN 3-88246-162-4.

\section{Heft 35}

Wolff, Armin; Gügold, Barbara (Hrsg.): Deutsch als Fremdsprache ohne Mauern. Beiträge der 19. Jahrestagung DaF 1991 in Berlin. 1. Auflage Regensburg 1993, VII, 299 Seiten, broschiert, DM 40,00/32,00*, ISBN 3-88246-165-9.

\section{Heft 36}

Müller-Küppers, Evelyn: Dependenz-/Valenz- und Kasustheorie im Unterricht Deutsch als Fremdsprache. Diss. Mainz 1989. 1. Auflage Regensburg 1991, III, 296 Seiten, broschiert DM 25,00/22,00*, ISBN 3-88246-168-3 (vergriffen).

\section{Heft 37}

Tütken, Gisela; Neuf-Münkel, Gabriele (Hrsg.): Schreiben im DaF-Unterricht an Hochschulen und Studienkollegs I: Forschungsergebnisse - Didaktische Konzeptionen - Übungsformen. Vorträge der Fachtagung DaF 1991. 1. Auflage Regensburg 1993, 277 Seiten, broschiert DM 34,00/ 27,00*, ISBN 3-88246-169-1.

\section{Heft 38}

Tütken, Gisela; Neuf-Münkel, Gabriele 
(Hrsg.): Schreiben im DaF-Unterricht an Hochschulen und Studienkollegs II: Texte ausländischer Studierender. Materialien der Fachtagung DaF 1991. 2. verbesserte Auflage Regensburg 1993, III, 150 Seiten, broschiert DM $28,00 / 22,50 *$, ISBN 388246-170-5.

\section{Heft 39}

Fiß, Sabine (Hrsg.): Deutschsprachige Fachkommunikation im universitären und außeruniversitären Bereich. Beiträge der Fachtagung DaF 1992. 1. Auflage Regensburg 1994, VII, 140 Seiten, broschiert DM 30,00/26,00*, ISBN 3-88246-178-0.

\section{Heft 40}

Wolff, Armin; Welter, Winfried (Hrsg.): Mündliche Kommunikation; Unterrichtsund Übungsformen DaF; Themen- und zielgruppenspezifische Auswahl von Unterrichtsmaterialien; Modelle für studien- und berufsbegleitenden Unterricht; DaF im Ausland. Beiträge der 20. Jahrestagung DaF 1992 in Münster. 1. Auflage Regensburg 1995, IV, 350 Seiten, broschiert DM 47,00/ 41,00*, ISBN 3-88246-180-2.

\section{Heft 41}

Albers, Hans-Georg (Hrsg.): Fort- und Weiterbildung von Lehrkräften für Deutsch als Fremdsprache. Beiträge der Fachtagung $\mathrm{DaF}$ 1993. 1. Auflage Regensburg 1995, IV, 122 Seiten, broschiert DM 32,00/ 28,00*, ISBN 3-88246-182-9.

\section{Heft 42}

Wolff, Armin; Köppel, Anette; SteinMeintker, Anneliese (Hrsg.): Autonomes Lernen. Lernpsychologie im Fremdsprachenunterricht. Deutsch als Fremdsprache im internationalen Kontakt. Qualitätskriterien für Sprachkurse DaF im außeruniversitären Bereich. Beiträge der 21. Jahrestagung DaF 1993 in Erlangen. 1. Auflage Regensburg 1996, 260 Seiten, broschiert DM 39,00/ 35,00, ISBN 3-88246-183-7.
Heft 43

Wolff, Armin; Schleyer, Walter (Hrsg.): Fach- und Sprachunterricht. Gemeinsamkeiten und Unterschiede. - Studiengänge Deutsch als Fremdsprache: Von der Theorie zur Praxis. Beiträge der 22. Jahrestagung DaF 1994 in Aachen. 1. Auflage Regensburg 1996, 480 Seiten, broschiert DM 49,00/43,00*, ISBN 3-88246-186-1.

\section{Heft 44}

Wolff, Armin; Blei, Dagmar (Hrsg.): DaF für die Zukunft. Eine Zukunft für DaF! Beiträge der 23. Jahrestagung DaF 1995 in Dresden. 1. Auflage Regensburg 1997, ISBN 3-88246-187-X (in Vorbereitung).

Heft 45

Ehlich, Konrad; Redder, Angelika (Hrsg.): »Schnittstelle Didaktik«. Empirische Untersuchungen zum DaF-Unterricht. 1. Auflage Regensburg 1997, 280 Seiten, broschiert DM 33,00/29,00*, ISBN 388246-189-6.

\section{Heft 46}

Wolff, Armin; Tütken, Gisela; Liedtke, Horst (Hrsg.): Gedächtnis und Sprachenlernen; Prozeßorientiertes Fremdsprachenlernen; Deutschlehrerausbildung in West- und Osteuropa; eine Deutsche Literatur - AutorInnen nichtdeutscher Muttersprache. Beiträge der 24. Jahrestagung DaF 1996 in Göttingen. 1. Auflage Regensburg 1997, 480 Seiten, broschiert DM 49,00/43,00*, ISBN 3-88246-190-X.

\section{Heft 47}

Wolff, Armin; Eggers, Dietrich (Hrsg.): Emotion und Kognition beim Fremdsprachenlernen; Lernen mit neuen Medien; Deutsch als Fremdsprache und die Attraktivität des Studien- und Lernortes Deutschland. Beiträge der 25. Jahrestagung $\mathrm{DaF}$ 1997 in Mainz. 1. Auflage Regensburg 1998, ISBN 3-88246-191-8 (in Vorbereitung). 